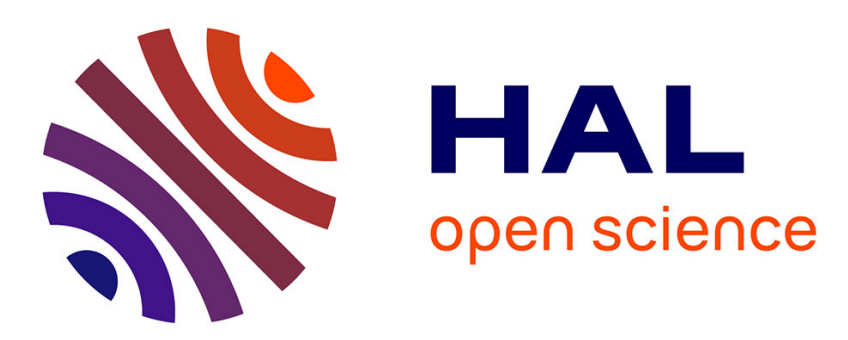

\title{
Les effets automatiques du parrainage télévisuel sur la marque: étude de la mésattribution de la familiarité, du transfert sémantique et de l'influence des émotions déclenchées par le programme
}

Didier Courbet

\section{To cite this version:}

Didier Courbet. Les effets automatiques du parrainage télévisuel sur la marque: étude de la mésattribution de la familiarité, du transfert sémantique et de l'influence des émotions déclenchées par le programme. Recherche et Applications en Marketing (French Edition), 2000, 15 (1), pp.39-61. sic_00001304v2

\author{
HAL Id: sic 00001304 \\ https://archivesic.ccsd.cnrs.fr/sic_00001304v2
}

Submitted on 26 Feb 2008

HAL is a multi-disciplinary open access archive for the deposit and dissemination of scientific research documents, whether they are published or not. The documents may come from teaching and research institutions in France or abroad, or from public or private research centers.
L'archive ouverte pluridisciplinaire HAL, est destinée au dépôt et à la diffusion de documents scientifiques de niveau recherche, publiés ou non, émanant des établissements d'enseignement et de recherche français ou étrangers, des laboratoires publics ou privés. 


\title{
Les effets automatiques du parrainage télévisuel sur la marque : étude de la mésattribution de la familiarité, du transfert sémantique et de l'influence des émotions déclenchées par le programme
}

\author{
Didier Courbet \\ Maître de conférences à l'Université de Nice-Sophia Antipolis * \\ Laboratoire de Psychologie Expérimentale et Quantitative
}

RÉSUMÉ

Grâce à une expérimentation, cette recherche étudie les effets non conscients du parrainage de deux programmes quasi identiques, mais de valences affectives différentes, sur l'attitude à l'égard d'une nouvelle marque. Les effets, enregistrés par tests indirects, sont indéniables pour le parrainage du programme gai : avec deux apparitions, celui-ci a un impact positif sur la marque. Le programme triste n'a eu aucun effet : l'intensité des émotions déclenchées aurait provoqué des pensées impertinentes empêchant de mémoriser la marque. Les résultats sont en accord avec le nouveau modèle de la mésattribution de la familiarité. Aucune trace d'un transfert sémantique automatique du programme sur l'image de la marque n'est observée.

Mots clés : parrainage télévisuel, parrainage, influence, télévision, publicité, affectif, émotions, cognitif.

\section{INTRODUCTION}

Le parrainage télévisuel consiste en un apport de ressources (le plus souvent financier) d'une entreprise ou d'une personne morale publique ou privée dans le but d'associer son nom, sa marque, son activité et ses réalisations à la diffusion d'un programme par une chaîne de télévision (e.g. Darty parrain de la météo sur F 2). Cette pratique est bien un type de parrainage des organisations selon le sens défini par Derbaix, Gérard et Lardinoit (1994) : le programme est considéré comme un événement socio-culturellement indépendant de l'organisation et financé directement ; le parrainage TV médiatise l'association programme-organisation (programme-marque) et réalise des objectifs de communication-marketing. En effet, la marque s'associe avec un programme pour bénéficier de certains de ses attributs. De plus, elle transmet un message, en dehors des écrans publicitaires, dans de courtes séquences de 6 secondes accolées aux pré- et postgénériques de l'émission. Dans ces séquences, le parrainage TV utilise à la fois des procédés de communication qui lui sont spécifiques (pour représenter l'association marque-programme par exemple) et des procédés empruntés à la création publicitaire (voir Courbet, 1999). Apparue, en France en 1984 sur Canal+, cette technique représente maintenant près d'un dixième des investissements en communication à la télévision ${ }^{1}$. Malgré les enjeux économiques, son processus d'influence sur les récepteurs et les effets sur la marque sont relativement méconnus.

* L'auteur peut être joint à l'Université de Nice, faculté des lettres et sciences humaines, 98 Bd E. Herriot, BP 3209, 06204 Nice cedex 3 ; mail : dcourbet@unice.fr

I. Source SECODIP. 
Après avoir effectué une première synthèse des théories actuelles expliquant le mode d'influence individuelle, l'objectif est de tester expérimentalement deux modèles : le modèle des transferts, actuellement dominant, et le modèle de la mésattribution de la familiarité, nouveau modèle d'influence construit à partir de la psychologie cognitive. Il s'agit, plus globalement, de mieux expliquer les effets cognitifs et affectifs du parrainage TV sur les représentations et les évaluations de la marque et, plus particulièrement, d'étudier l'impact des émotions induites par le programme.

\section{CADRE THÉORIQUE DE L'INFLUENCE DU PARRAINAGE ET DU PARRAINAGE TV}

Les recherches sur l'influence du parrainage sportif, bien développées dans les pays francophones, ont essentiellement consisté en l'établissement d'équations structurelles ${ }^{2}$ et de réseaux mettant en relation des variables explicatives et des variables expliquées (Giannelloni, 1990, 1993 ; Walliser, 1994, 1996 ; Lardinoit, 1996). Malgré l'apport considérable de ces travaux, ceux-ci ne permettent pas de modéliser en détail l'aspect dynamique des traitements des informations effectués par le récepteur. Nous nous intéressons ici davantage aux théorisations des processus psychologiques : quelles sont les différentes étapes de l'élaboration des représentations de la « marqueparrain »? Quelles sont les étapes qui conduisent à son évaluation?

Le modèle le plus souvent avancé, tant par les praticiens que par les chercheurs, est celui du transfert d'éléments propres à l'événement parrainé sur l'image de la marque (Ganassali et Didellon, 1996). Nous proposons une autre explication de l'influence psychologique : le modèle de la mésattribution de la familiarité.

\section{Le modèle des transferts et ses limites}

Selon ce modèle, deux types de transferts sont possibles. Avec un transfert affectif (voir notamment

2. Les psychologues utilisent, quant à eux, le terme «structurales».
Janiszewski et Warlop, 1993), les réactions émotionnelles déclenchées, tout d'abord, par le seul programme vont être également déclenchées, ensuite, par la vision de la marque-parrain seule. Si, par exemple, le programme est évalué positivement, la marque le sera ensuite également. Un transfert sémantique (McCracken, 1989) permettrait à la marque de bénéficier d'attributs de l'entité parrainée. Ainsi, ces attributs pourraient être transférés du programme sur le parrain. Même si la littérature est floue sur ce point, il semblerait que les récepteurs ne soient pas conscients de ces mécanismes ; les résultats de ces transferts pourraient cependant se constater avec des mesures verbales. Les supports théoriques et empiriques de ce modèle nous paraissent cependant très minces. Pour expliquer ces deux transferts, certains chercheurs en marketing font appel au mythe encore bien trop tenace du conditionnement classique. Il convient de rappeler que celui-ci ne constitue nullement une explication théorique mais un simple protocole expérimental grâce auquel les psychologues constatent des effets d'apprentissage. Il faut ensuite expliquer en détail par quels processus s'opèrent les associations et les éventuels transferts (pour une large discussion, voir Courbet, 1997, pp. 401-413 ; pour une présentation plus détaillée des modèles d'influence expliqués ci-dessous, voir Courbet, 1999).

\section{Le modèle de la mésattribution de la familiarité}

Ce modèle, construit à partir de récentes recherches en psychologie cognitive, part du principe que le télespectateur n'accorde pas une forte attention à la séquence de parrainage TV. Il ne traite pas le message de manière complexe. Pour illustrer les deux étapes qui constituent ce modèle, prenons le cas d'une nouvelle marque parrainant un programme.

$-1^{\text {te }}$ étape : Au cours de la première exposition, les traitements (vision ou audition) faiblement élaborés (rapides et/ou peu approfondis) de cette marque forment, dans la mémoire du téléspectateur, des représentations d'un niveau uniquement structural (figuratif, e.g. le dessin du logotype), sans contenu sémantique ni attribut précis et sans charge affective marquée. Ensuite, plus le sujet est exposé à la marque, plus il va décoder rapidement les signes la représentant. Cette différence de rapidité serait inférieure à la seconde : la marque lui semble, à chaque nouveau contact, un peu 
plus familière. Selon la théorie psychologique néoconnexionniste, cette vague familiarité manifesterait une fluidité des réseaux cognitifs (voir McClelland et al., 1995 ; McClelland et Rumelhart, 1988, pour une vue d'ensemble).

- $2^{\mathrm{e}}$ étape : Lorsque le sujet aperçoit la marque (ou ses produits), par exemple sur le lieu de vente, il ressentirait, tout d'abord et immédiatement, une vague sensation de l'avoir déjà vue. Puisque les représentations qu'il a en mémoire ne sont pas sémantiques mais uniquement structurales, le sujet n'a ni image de la marque ni attitude à son égard. En outre comme les traitements au cours de la première étape n'ont pas été élaborés, sa mémoire ne lui permet pas de savoir où il l'a déjà vue : il a oublié qu'il a été exposé à des messages à la télévision. Ne pouvant être attribuée à sa réelle source, cette vague impression de familiarité serait alors spontanémént mésattribuée (faussement attribuée), de manière automatique (sans conscience), à des caractéristiques propres à la marque. Il émettrait, par exemple, l'hypothèse qu'elle est une bonne marque : c'est l'évaluation affective qui bénéficie ici de la mésattribution. En psychologie, les supports théoriques et empiriques d'un tel principe sont solides : on le retrouve en effet, sous une forme légèrement différente, dans les recherches sur les erreurs d'attribution et les apprentissages non conscients (pour des revues, voir Meulemans, 1998 en psychologie cognitive ; Bornstein et Pittman, 1992 en psychologie sociale cognitive).

\section{Hypothèses et objectifs principaux}

Le modèle du transfert affectif postule qu'une marque est mieux évaluée lorsqu'elle parraine un programme déclenchant des réponses affectives positives que lorsqu'elle parraine un programme déclenchant des réactions négatives. Or nous estimons que le modèle de la mésattribution de la familiarité, dont les supports théoriques sont plus solides, explique mieux le processus d'influence et les effets. Nous nous attendons à ce que la réexposition à une marque-parrain provoque, avant tout, une impression de familiarité (hypothèse $\mathrm{H}_{1}$ ). Nous postulons également l'absence d'effet spécifique des émotions déclenchées par le programme sur les représentations et les évaluations de la marque. Quel que soit l'état affectif (négatif ou positif) provoqué par le programme, la fluidité cogni- tive se manifeste de la même manière : l'impression de familiarité apparait (hypothèse $\mathrm{H}_{2}$ ). Lorsque celle-ci est présente, elle est mésattribuée : la marque est alors mieux évaluée ; lorsque elle n'est pas présente, la marque n'est pas mieux évaluée (hypothèses $\mathrm{H}_{3}$ ). De plus, nous cherchons à réfuter le modèle du transfert sémantique sans conscience. Sans souvenir clair du message et du parrainage TV, l'image de la marque et de ses produits ne peuvent pas bénéficier du transfert d'attributs (hypothèse $\mathrm{H}_{4}$ ). Les hypothèses opérationnelles figurent dans le tableau 1 . Outre ces hypothèses, l'objectif est d'expliquer les processus d'influence du parrainage TV sur la marque en étudiant particulièrement l'impact des émotions déclenchées par le programme parrainé.

\section{MÉTHODE}

\section{Minimisation des biais grâce à l'enregistrement des effets automatiques}

Au niveau méthodologique, l'étude de l'influence du parrainage doit éviter un obstacle de taille : le biais de conscience de la demande. Après la phase d'exposition aux séquences, l'expérimentateur questionne le sujet sur la marque. Ce biais apparait lorsque le sujet se remémore, avant de répondre, la séquence et le programme parrainé. C'est à ce moment précis qu'il construit « artificiellement » l'image de la marque en extrayant des caractéristiques appartenant au programme (« la marque parraine du sport, elle voudrait donc qu'on la considère dynamique »). Ainsi, l'étude risque fort de montrer l'existence d'un soi-disant transfert ; or ce n'est rien d'autre que le résultat d'un traitement conscient biaisant l'influence réelle de l'action. Nous construisons le matériel et un scénario expérimental pour empêcher ce biais. Le sujet ne doit pas se remémorer consciemment (de manière verbalisable) le programme au moment où il est questionné sur la marque-parrain. On évalue ainsi les effets automatiques du parrainage TV sur la marque. Pour éviter la mémorisation du lien marque-programme, le nombre d'apparitions de la marque doit donc être limité (dans notre expérimentation : deux expositions). 
Tableau 1. - Hypothèses de la recherche

\begin{tabular}{|c|c|}
\hline Codage: & Hypothèses \\
\hline $\mathrm{H} 1$ & $\begin{array}{l}\text { La réexposition à la marque Calmin provoque une impression de familiarité : les différences sont signifi- } \\
\text { catives entre, d'une part, les groupes expérimentaux exposés (quel que soit le programme), et les non- } \\
\text { exposés, d'autre part. }\end{array}$ \\
\hline $\mathrm{H} 2$ & $\begin{array}{l}\text { La différence des valences affectives des programmes parrainés ne provoque pas de différence dans les } \\
\text { impressions de familiarité. Les résultats sont identiques entre les groupes exposés au programme gai et ceux } \\
\text { exposés au programme triste. }\end{array}$ \\
\hline $\begin{array}{l}\mathrm{H} 3 \text { : } \\
\text { - soit } \mathrm{H} 3 \mathrm{a} \\
\text { - soit } \mathrm{H} 3 \mathrm{~b}\end{array}$ & $\begin{array}{l}\text { H3a : Si H1 et H2 sont validées }{ }^{1} \text {, les groupes exposés aux programmes gai et triste ont des évaluations } \\
\text { affectives de la marque plus favorables que celles des groupes non exposés. Il existe des différences } \\
\text { significatives sur un ou plusieurs attribut(s) marketing, entre: } \\
\text { - le groupe exposé au programme gai et le groupe non exposé, } \\
\text { - le groupe exposé au programme triste et le groupe non exposé. } \\
\text { Par opposition, aucune différence n'existe entre les effets obtenus par les programmes gai et triste sur ces } \\
\text { évaluations. } \\
\text { - H3b : } \mathrm{Si} \text { H1 et H2 sont invalidées }{ }^{1} \text {, nous postulons l'absence de telles différences. }\end{array}$ \\
\hline $\mathrm{H} 4$ & $\begin{array}{l}\text { Absence de différence dans les scores sur les attributs transférés des programmes, canal et contexte } \\
\text { entre, d'une part, les groupes expérimentaux exposés (quel que soit le programme) et, d'autre part, les non- } \\
\text { exposés (groupe contrôle). }\end{array}$ \\
\hline
\end{tabular}

1 Dans le modèle de la mésattribution de la familiarité, les deux étapes, représentées respectivement par $\mathrm{H}_{1}$ et $\mathrm{H}_{2}$, d'une part, et $\mathrm{H}_{3}$, d'autre part, sont indépendantes. Il faut donc prévoir deux possibilités $\left(\mathrm{H}_{3}\right.$ a et $\left.\mathrm{H}_{3} \mathrm{~b}\right)$.

\section{Conception et test du matériel}

Deux programmes de qualité professionnelle, intitulés «Souvenirs du passé », d'une durée de $4 \mathrm{mn} 06 \mathrm{~s}$, sont conçus et réalisés. Les éléments du champ (la comédienne et le décor), les techniques de filmage, le cœur du scénario sont, pour les deux, parfaitement identiques. Une seule caractéristique les différencie : la valence affective d'un même souvenir exprimé par une patiente chez son psychologue est rendue soit triste soit gaie par le contenu du discours et quelques expressions du visage de l'actrice. L'objectif est ici d'isoler, le plus soigneusement possible, l'effet des émotions ${ }^{3}$. Le matériel vise à faire ressentir, dans chaque groupe expérimental, des émotions différentes sans que d'autres facteurs ne varient. Afin de

3. En fait, très peu d'expériences sur la dimension affective en psychologie sociale et en maketing se préoccupent de l'isolement strict des variables. mieux contrôler les représentations préalables de la marque, nous créons une pseudomarque-parrain : «Calmin » ${ }^{4}$. Prégénérique, postgénérique et séquences de parrainage sont identiques pour les deux programmes (voir annexe).

Une pré-enquête a été réalisée avec trois méthodes : analyse de contenu effectuée par un spécialiste de sémiologie filmique (les résultats sont synthétisés dans le tableau 2); entretiens individuels (six sujets, durée $20 \mathrm{mn}$ ) et entretiens de groupe (6 sujets, durée $30 \mathrm{mn}$ ). Elle a confirmé, d'une part, que les thèmes programmatiques sont tous bien décodés et identiques dans les deux programmes. Elle a montré, d'autre part, que chaque programme provoque les réactions affectives recherchées. Enfin, la marque, inconnue et bien lisible, peut concerner de nombreux secteurs de produits.

4. L'utilisation d'une marque connue avec une image déjà précise aurait constituée de nouvelles sources de variations difficilement maitrisables. 
Tableau 2. - Comparaison des deux programmes

\begin{tabular}{|c|c|c|}
\hline Criteres de comparaison: & $\begin{array}{l}\text { Contenu du programine a triste }>\text {. } \\
\text { generant des réactions affectives negatives }\end{array}$ & $\begin{array}{l}\text { Contenu du programme i gat in } \\
\text { génerant des réactions affectives positives }\end{array}$ \\
\hline $\begin{array}{l}\text { - Personnage principal (identique } \\
\text { dans les deux programmes) } \\
\text { - Personnalité-humeur }\end{array}$ & $\begin{array}{l}\text { - femme, } 30 \text { ans, style « } B C B G » \\
\text { - triste, déprimée }\end{array}$ & $\begin{array}{l}\text { - femme, } 30 \text { ans, style « BCBG» } \\
\text { - gaie, exubérante, ironique }\end{array}$ \\
\hline $\begin{array}{l}\text { Contexte socio-physique de la } \\
\text { scène (identique dans les deux } \\
\text { programmes) }\end{array}$ & $\begin{array}{l}\text { - psychothérapie (patiente allongée sur un } \\
\text { divan) } \\
\text { - rares regards adressés au psychothérapeute } \\
\text { (monologue) } \\
\text { - cadre physique sobre }\end{array}$ & $\begin{array}{l}\text { - psychothérapie (patiente allongée sur un } \\
\text { divan) } \\
\text { - rares regards adressés au psychothéra- } \\
\text { peute (monologue) } \\
\text { - cadre physique sobre }\end{array}$ \\
\hline $\begin{array}{l}\text { - Thèmes des programmes } \\
\text { (identiques dans les deux } \\
\text { programmes) } \\
\text { - Affects associés }\end{array}$ & - évocation de souvenirs de famille & - évocation de souvenirs de famille \\
\hline
\end{tabular}

\section{Procédures, sujets et plan d'expérience}

Le scénario expérimental et la forte déperdition d'effectif causée par les particularités de la procédure nous conduisent à choisir des étudiants ${ }^{5}$. La préenquête et les tests des questionnaires ont été réalisés sur 65 étudiants dont le profil est identique à celui des sujets expérimentaux. Pour obtenir finalement un échantillon exploitable de 136 sujets, l'expérience a porté sur 225 sujets (forte déperdition entre les deux phases expérimentales). Elle a été effectuée deux fois, à chaque fois sur une population différente. La distribution dans les groupes (expérimentaux, de contrôle et de pré-enquête), par ordre alphabétique, est celle de l'institution. Elle se rapproche - sans l'être totalement - d'une distribution aléatoire ${ }^{6}$. Les groupes sont donc quasi équivalents. Afin de minimiser les biais dus aux éventuelles communications des sujets entre eux, le silence au moment des réponses aux questionnaires a été demandé et vérifié. La présence et l'absence du traitement expérimental ont été affectées au hasard dans les groupes :

- Phase T1 (divisée en 2 phases : $t 1$ et t' 1 ). En $t 1$ : exposition de chacun des deux groupes expérimen-

5. IUT de l'Université de Nancy-II, départements Informatique et Information et communication.

6. Nous avons vérifié qu'aucun groupe ne comporte davantage de patronymes reliés à des caractéristiques culturelles ou ethniques. taux à un des deux programmes TV. L'exposition a lieu au début d'un des cours habituels des étudiants, dans leur salle (conditions d'exposition identiques pour tous les groupes). En t' 1 : enregistrement, immédiatement après, des états affectifs et des évaluations du seul programme (durée totale de $\mathrm{T} 1: 20 \mathrm{mn}$ ).

- Phase T2 : 7 jours après l'exposition, enregistrement des réactions à la marque Calmin sans que les sujets n'établissent de lien avec l'exposition. Une secrétaire de l'IUT, arrive « à l'improviste » au début d'un cours. Elle tend à l'enseignant comparse une pile de questionnaires en indiquant qu'il s'agit de « l'enquête annuelle de l'IUT »: une entreprise lorraine désire tester trois logotypes et noms de marques nouveaux afin de sélectionner les plus adaptés pour des nouveaux produits. Un premier nouveau logotype présent uniquement pour la crédibilité du scénarioest présenté. Les sujets répondent ensuite au premier questionnaire sur le logotype. Un deuxième logotype est présenté (notre marque Calmin), suivent ensuite les réponses au second questionnaire. Un troisième logotype - créé également pour cette expérience est montré, puis la phase de réponse au troisième questionnaire (seul le second questionnaire nous intéresse). Une consigne importante est donnée par l'entreprise dans une lettre : les réponses doivent être exprimées individuellement et sans remarques à haute voix (durée : $20 \mathrm{mn}$ ). Tous ces questionnaires sont ensuite ramassés. 


\begin{tabular}{|c|c|c|c|c|}
\hline & \multicolumn{2}{|r|}{ T1 } & \multirow{2}{*}{$\begin{array}{c}\text { T2 } \\
7 \text { jours après } \\
\text { (questionnaire } \\
\text { sur les } \\
\text { marques) }\end{array}$} & \multirow{2}{*}{$\begin{array}{c}\mathrm{T} 3 \\
\text { immédiatement après } \\
\mathrm{T} 2 \\
\text { (questionnaire post- } \\
\text { expérimental }\end{array}$} \\
\hline & $\begin{array}{c}\mathrm{tl} \\
\text { Exposition }\end{array}$ & $\begin{array}{c}\text { t'l } \\
\text { immédiatement } \\
\text { après exposition } \\
\text { (questionnaire sur } \\
\text { le programme) }\end{array}$ & & \\
\hline $\begin{array}{l}\text { Parrainage pré et postgénériques d'un } \\
\text { programme provoquant des réactions } \\
\text { affectives positives (progr. "gai"). } \\
2 \text { groupes : GI gai et GE gai }\end{array}$ & $\mathrm{X}$ & Y1 & $Y^{\prime} 1$ & Y'1 \\
\hline $\begin{array}{l}\text { Parrainage pré et postgénériques d'un } \\
\text { programme provoquant des réactions } \\
\text { affectives négatives (progr. "triste") } \\
2 \text { groupes : GI triste et GE triste }\end{array}$ & $\mathrm{X}$ & $\mathrm{Y} 2$ & Y'2 & Y'2 \\
\hline $\begin{array}{l}\text { Groupe contrôle } \\
2 \text { groupes : GCI et GCE }\end{array}$ & $\varnothing$ & $\varnothing$ & $Y^{\prime} 3$ & $\varnothing$ \\
\hline \multicolumn{5}{|l|}{ Codage des groupes: } \\
\hline $\begin{array}{l}\text { G : groupe expérimental ; GC : gr } \\
\text { I : étudiants du département infor }\end{array}$ & $\begin{array}{l}\text { pe contrôle ; } \\
\text { atique ; }\end{array}$ & & & \\
\hline
\end{tabular}

Figure 1. - Plan d'expérience

- Phase T3 (immédiatement après T2) : la secrétaire distribue le questionnaire post-expérimental où le réel but de l'expérience est indiqué (durée 5 minutes).

Dans le plan d'expérience à groupes quasi équivalents, les sujets du groupe contrôle (GC) n'ont été exposés ni à la marque ni au matériel expérimental : seule la phase T2 est réalisée ${ }^{7}$ (voir figure 1).

\section{Mesures pour s'assurer que les conditions expérimentales sont bien remplies}

\section{- Mesures des états affectifs avant-après et des évaluations affectives des programmes (phase t'l)}

On compare, tout d'abord, l'état affectif des sujets juste avant la réception des programmes à l'état affectif dans lequel ils se trouvent juste après à l'aide de mesures d'émotions. Pour s'assurer que les évolutions

7. Les jours et les conditions de passation sont identiques pour tous les groupes dont les résultats seront statistiquement comparés. sont bien attribuables aux programmes, une deuxième série d'échelles mesure, en outre, les évaluations affectives des programmes faites par les sujets ${ }^{8}$.

Les premières mesures s'inspirent des échelles américaines the PANAS Scales de Watson, Clark et Tellegen (1988) d'une part, et, d'autre part, des échelles en langue française de Derbaix (1993). Les enregistrements s'effectuent avec des échelles d'autoévaluation unipolaires à 5 échelons. Les sujets répondent à 25 items émotionnels par un chiffre de 0 (l'émotion en question «n'est pas ressentie du tout ») à 4 (« très ») ${ }^{9}$. Le même dispositif de présentation et de réponse est proposé pour l'état « juste avant le

8. Il s'agit de faire en sorte que le téléspectateur ressente véritablement les émotions pertinentes et non uniquement de s'assurer que le programme-inducteur est évalué par les adjectifs pertinents. Juste avant l'apparition de la marque, le sujet est déjà dans un certain état affectif (l'humeur dans laquelle il se trouve, l'excitation provoquée par l'expérience...). Un programme qualifié de «triste » doit réellement provoquer de la tristesse chez le récepteur. Les mesures doivent donc considérer l'état affectif du sujet avant le traitement de la séquence puis le comparer avec l'état affectif juste après.

9. Seuls les résultats aux items les plus représentatifs sont traités ; voir tableaux 6 et 7 . 
début du programme » et « juste après la fin du programme ».

La deuxième série d'échelles enregistre des jugements sur le programme. Les items d'évaluation sont construits à partir d'adjectifs issus, d'une part, de la terminologie classiquement utilisée pour juger un film, de la théorie psycholinguistique des émotions (Bonis, 1996), d'autre part, et, enfin, des résultats de la pré-enquête. Une série de 12 adjectifs est proposée à la suite de la question «si vous deviez juger le programme vous diriez qu'il est ${ }^{10}$. Le sujet attribue à chacun un chiffre de 0 à 5 . Un ancrage verbal minimal étiquette uniquement les deux chiffres extrêmes, respectivement par « pas du tout » ou « extrêmement ».

\section{- Questionnaire post-expérimental pour s'assurer que la marque-parrain n'a pas été reconnue (phase T3)}

Seuls nous intéressent les effets automatiques des actions de parrainage TV enregistrés en l'absence de souvenir de l'exposition au matériel expérimental. Nous devons nous assurer qu'aucun lien conscient entre la marque sur laquelle on demande au sujet de réagir et le programme passé la semaine précédente n'a été établi. Le sujet doit uniquement récupérer en mémoire la trace due aux effets de l'exposition sept jours avant : il ne doit pas construire artificiellement des représentations sur la marque au moment où on lui demande de remplir le questionnaire. On cherche ainsi l'impact réel et direct de l'action. Inspiré d'expériences en psychologie sociale, un questionnaire post-expérimental enregistre cette éventuelle reconnaissance. Les données issues des sujets ayant reconnu la marque-parrain ont été éliminées (voir résultats plus loin).

\section{- Mesures et opérationnalisation des deux modèles testés}

Les processus que nous cherchons à mettre en évidence échappent à la conscience des sujets. Pour enregistrer ces automatismes cognitifs, nous utilisons systématiquement des tests indirects par choix forcés issus de la psychologie de la mémoire et de l'apprentissage implicite (voir Stadler et Frensch, 1998 ; Nicolas et Perruchet, 1998 ; Perruchet et Nicolas, 1998 ; Perruchet, 1980). Ils permettent, tout d'abord, de

10. Les adjectifs figurent dans le tableau 5. mettre en évidence les effets de l'exposition sans que le sujet ait souvenir de l'épisode d'apprentissage. L'absence de conscience de la demande évite, ensuite, la mise en place de stratégies conscientes où le sujet, soit biaiserait sa réponse, soit reconstruirait artificiellement l'image de la marque à partir du programme. Il n'y a, en outre, pas de «bonnes ou mauvaises réponses » : le sujet ne craint pas de faire une erreur ou de devoir justifier sa réponse. Enfin, on force le choix : pour enregistrer la réponse, six échelons obligent à prendre position d'un coté ou de l'autre de l'échelle ; l'étiquette « ne sait pas » ne figure pas ${ }^{11}$. Ces tests peuvent ainsi enregistrer une évaluation à peine polarisée ainsi que la moindre tendance à s'orienter vers un côté de l'échelle. Ce type de mesures, très sensibles, permettraient de mettre en évidence les traces d'une influence alors que le sujet est incapable, consciemment, de rappeler ou reconnaître la marque-parrain. La récupération de la trace mnésique est donc bien automatique (pour la psychologie cognitive, il s'agit d'automatismes post-cablés ; voir Camus, 1996).

\section{- Mesures concernant le modèle de la mésattribution de la familiarité}

Ce modèle, en deux processus indépendants, nécessite deux séries de mesures.

\section{- Les mesures de la familiarité}

L'objectif est d'enregistrer, avec un outil verbal, l'impression de familiarité envers la marque expérimentale, alors que le sujet n'a plus souvenir de l'exposition télévisuelle de la semaine précédente. La soi-disant étude commerciale de T2 permet de ne demander qu'un « avis personnel », sans bonne ou mauvaise réponse. Ainsi sont minimisées les stratégies de « gain ou perte », biaisant souvent la réponse des sujets dans les expériences sur la mémorisation. Quatre séries de deux items sont conçues et débutent le questionnaire. Le contenu des items est justifié par les théories sur le traitement cognitif de l'information sensorielle. Les deux premières séries d'items mesu-

11. Seules deux étiquettes verbales ancrent chaque extrémité de l'échelle. En effet, le langage est peu adapté pour discerner de manière valide les différentes modalités et les étiquettes numériques obligent, de manière peu satisfaisante, soit à fixer un « 0 » ou des scores négatifs, soit une hiérarchisation numérique qui pourraient influencer le répondant. 
Tableau 3. - Items d'évaluation de la familiarité et variables de marque

\begin{tabular}{|c|c|c|}
\hline 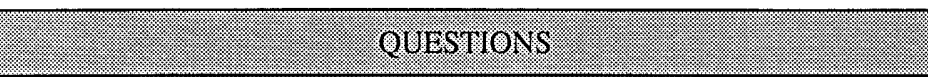 & REPONSES & COD.AE \\
\hline $\begin{array}{l}\text { Évaluation de la familiarité : } \\
\text { Avez-vous l'impression même extrêmement vague que : } \\
\text { 1) la marque existe déjà ou a déjà existé (c'est votre première impres- } \\
\text { sion qui nous intéresse)? } \\
\text { 2) vous avez déjà vu ou entendu la marque avant aujourd'hui? } \\
\text { 3) le nom de la marque (uniquement le son) ressemble, sans être } \\
\text { rigoureusement le même, à celui d'une marque qui existe déjà (que } \\
\text { vous sachiez ou non le nom auquel il ressemble, répondez unique- } \\
\text { ment en fonction de votre impression)? } \\
\text { 4) le dessin de la marque ressemble, sans être rigoureusement le même, } \\
\text { à celui d'une marque qui existe déjà (que vous sachiez ou non le nom } \\
\text { de cette marque, répondez uniquement selon votre impression)? }\end{array}$ & $\begin{array}{l}\text { «Cochez oui si votre impression } \\
\text { est extrêmement vague, très } \\
\text { vague, vague ou plus forte. } \\
\text { Cochez non dans les autres cas » }\end{array}$ & $\begin{array}{l}\text { 3) Copinom } \\
\text { 4) Copidess }\end{array}$ \\
\hline $\begin{array}{l}\text { Variables d'évaluation affective : } \\
\text { - La marque est extr. plaisante (codage réponse : 1) vs déplaisante } \\
\text { (codage réponse : } 6 \text { ) } \\
\text { - La marque est extr. bien } v s \text { mal choisie } \\
\text { - La marque donne aux produits une extr. bonne } v s \text { mauvaise image } \\
\text { Attributs marketing: } \\
\text { - La marque donne aux produits une image extr. haut } v s \text { bas de gamme } \\
\text { - La marque convient davantage à des produits d'invention extr. récente } \\
\text { vs ancienne }\end{array}$ & $\begin{array}{l}\text { - } 6 \text { échelons } \\
\text { - étiquettes verbales aux extré- } \\
\text { mités } \\
\text { - pas d'étiquette numérique }\end{array}$ & $\begin{array}{l}\text { - plaisant } \\
\text { - choix } \\
\text { - imagprod }\end{array}$ \\
\hline
\end{tabular}

rent la familiarité du stimulus pris dans sa globalité : elles portent sur la forme générale des signes de marque, sans distinction analytique. Les deux items suivants demandent un traitement analytique des signes de marque et mesurent la familiarité propre à différentes parties du stimulus : d'abord le nom (son), puis le dessin de la marque. Tout comme les traitements des signes linguistiques et des signes non linguistiques, les traitements des informations sensorielles (vision des signes de marque et audition du nom - son -) sont, en effet, différents. De plus, avec les libellés, les sujets n'ont pas à craindre qu'on exige d'eux l'indication d'une réponse plus précise ( ${ }^{\text {re }}$ partie du tableau 3 ). Les quatre items sont présentés selon l'ordre qui donnerait les meilleurs résultats : le premier devrait être le plus sensible, le dernier le moins sensible. Les variables sont enregistrées par un test implicite qui mesure l'absence $v s$ la présence de cette impression ${ }^{12}$.

12. Au niveau théorique, cette variable dichotomique est justifiée par le modèle de la dissociation des processus (Jacoby, 1991; Jacoby, Toth et Yonelinas, 1993). Il postule l'existence d'une loi du « tout ou rien » dans la récupération : « on a ou on n'a pas l'impression de connaître un objet spécifique ».
- Mesures de « variables de marque » ${ }^{13}$, objets de la mésattribution de la familiarité

Lorsqu'une vague impression de familiarité se manifeste, à quelles variables de marques peut-elle être mésattribuée ? On propose plusieurs variables marketing dont on enregistre les scores par la technique de tests indirects par choix forcé. Nous mesurons, tout d'abord, l'éventuelle mésattribution à des variables d'évaluation affective. La première échelle évalue le caractère plaisant $v s$ déplaisant de la marque. La seconde évalue le choix des signes de marque (bien $v s$ mal choisis). La troisième mesure, enfin, l'image que la marque donnerait aux produits (bonne vs mauvaise). Nous mesurons, ensuite, l'éventuelle mésattribution à deux attributs marketing, non mis en valeur par l'opération ou dans le programme. Les consommateurs utilisent fréquemment ces attributs pour évaluer une marque. Parce qu'ils sont fortement accessibles (prégnants en mémoire), ils pour-

13. Nous appelons «variables de marque», les représentations et les évaluations de la marque. 
raient plus facilement faire l'objet de la mésattribution. Le premier attribut est la hauteur perçue de la gamme des produits (ou des services). Le second est l'âge perçu des produits ( $2^{\mathrm{e}}$ partie du tableau 3 ).

\section{- Mesures concernant le transfert sémantique automatique}

La démarche de recherche suivie est inhabituelle : nous construisons un dispositif d'observation très large afin de tenter d'enregistrer le moindre effet qu'un transfert sémantique automatique pourrait expliquer. En effet, selon ce modèle, la mémoire du téléspectateur pourrait associer à la représentation de la marque des éléments perçus au même moment que la séquence de parrainage. Dans la séquence fabriquée, rien n'est précisé sur le secteur de produit du parrain ou l'image type des consommateurs. Nous cherchons donc dans le contenu du programme mais également dans le contexte socio-physique de réception du message télévisuel des attributs sémantiques qui pourraient être transférés sur les représentations de la marque et encodés avec ses produits (e.g. la marque parrainant un programme où le seul personnage est une femme pourrait être encodée comme étant destinée aux femmes ; l'item est alors : « la marque est extrêmement adaptée vs extrêmement inadaptée aux produits uniquement destinés aux femmes »).

\section{- Les attributs transférés de la thématique} du programme

1) Les attributs relatifs à l'image type du consommateur de la marque. L'actrice, âgée d'une trentaine d'années, pourrait représenter le consommateur-type. Selon le programme, elle est soit gaie, soit triste. Trois variables sont transférées du programme : le sexe (codé « femme », « homme »), l'âge (4 items) et l'humeur du consommateur-type (codé « affectci »). L'humeur des consommateurs-types est évaluée par un attribut bipolaire « gaie $v s$ triste ».

2) Les attributs relatifs à la valence affective des occasions de consommation. Les occasions de consommation des produits de la marque sont scindées en deux items unidimensionnels : les occasions gaies et occasions tristes.

3) Les attributs relatifs aux secteurs de produits de la marque. Les thèmes programmatiques prégnants sont transformés en secteurs de produits particuliers.
Nous demandons si le sujet considère la marque adaptée ( $v s$ inadaptée, même échelle que précédemment) à différents secteurs de produits ayant un lien avec le thème du programme (tableau 4 ).

- Les attributs relatifs aux secteurs de produits transférés du contexte de réception et du canal de transmission.

Lors de la réception, ces facteurs sont au moins aussi sensoriellement et sémantiquement prégnants que le contenu du programme. Ils sont donc susceptibles d'être transférés (e.g. la marque, ayant été vue pour la première et seule fois à la $T V$, pourrait être associée en mémoire avec un téléviseur ${ }^{14}$ ). Nous demandons si le sujet considère la marque adaptée $v s$ inadaptée à deux secteurs de produits (tableau 4).

\section{RÉSULTATS ${ }^{15,16}$}

Avant de décrire les résultats concernant les deux modèles, nous montrons que les conditions expérimentales, indispensables pour tester les hypothèses, sont bien mises en place.

\section{Le bon établissement des conditions expérimentales}

\section{- Une absence de reconnaissance de la marque-parrain effective}

Les très faibles scores de reconnaissance montrent que les étudiants ont, dans leur grande majorité, réellement été « piégés ». Les données issues de quinze

14. Certains supports théoriques pourraient être trouvés dans les modèles mémoriels de l'encodage contextuel de Tulving (1983, 1985). Il convient donc de tester l'hypothèse selon laquelle un individu encode avec la marque un élément prégnant (« indice ») propre au contexte de réception et présent au même moment que la marque.

15. IUT de Nancy-II, les groupes issus du département Informatique sont codés $\mathrm{GI}$ pour les groupes expérimentaux et $\mathrm{GCI}$ pour le groupe contrôle ; les groupes issus du département Information et communication seront codés GE pour les groupes expérimentaux et GCE pour le groupe contrôle.

16. Pour certains tests, le seuil de risque, bilatéral dans les tableaux bruts, devient unilatéral lorsqu'un sens est fixé dans les hypothèses. 
Tableau 4. - Items relatifs aux secteurs de produits ; leur lien avec l'action de parrainage

\begin{tabular}{|c|c|c|c|}
\hline $\begin{array}{l}\text { SECTELRS DE PRODUITS } \\
\text { DEIIA MAROUE }\end{array}$ & $\begin{array}{l}\text { IIEN A VEC I ACTION } \\
\text { DE PARRAINAGE }\end{array}$ & het & CODAGE \\
\hline $\begin{array}{l}\text { Sect. transférés des programmes : } \\
\text { - des cotillons et accessoires de fête } \\
\text { - des jouets } \\
\text { - une association de soutien pour femme } \\
\text { en détresse morale } \\
\text { - un service funéraire et d'inhumation } \\
\text { - une collection de cassettes vidéo sur la vie } \\
\text { quotidienne dans les années } 60-70\end{array}$ & $\begin{array}{l}\text { Cotillons lancés dans le programme gai } \\
\text { Valence affective positive. } \\
\text { L'actrice évoque son passé de } \\
\text { fillette. Valence affective positive. } \\
\text { La patiente recherche une aide chez } \\
\text { le psychothérapeute Valence affective } \\
\text { négative. } \\
\text { Le thème de la mort est également } \\
\text { saillant. Valence affective négative. } \\
\text { La patiente raconte des événements } \\
\text { quotidiens qui ont eu lieu pendant cette } \\
\text { période. }\end{array}$ & $\begin{array}{l}6 \text { échelons } \\
\text { Extr. inadapté } \\
\text { (codé 1) vs } \\
\text { extr. inadapté } \\
\text { (codé 6) }\end{array}$ & $\begin{array}{l}\text { - cotillon } \\
\text { - jouets } \\
\text { - detresfe } \\
\text { - funéraire } \\
\text { - vidéo } 60\end{array}$ \\
\hline $\begin{array}{l}\text { Sect. de produits transférés du contexte } \\
\text { de réception et du canal : } \\
\text { - des accessoires scolaires } \\
\text { - du matériel TV-HIFI }\end{array}$ & $\begin{array}{l}\text { Le contexte socio-physique de l'exposi- } \\
\text { tion expérimentale est une salle de cours. } \\
\text { La marque est vue pour la première } \\
\text { et seule fois à la télévision. }\end{array}$ & & - TV HIFI \\
\hline
\end{tabular}

sujets ayant reconnu la marque-parrain ont été éliminées (soit un échantillon exploitable de 136 sujets). Les effets des deux actions de parrainage TV seront étudiés sans biais de conscience de la demande, en réelle absence de souvenirs de l'exposition et de l'action de parrainage elle-même. Les mesures seront donc effectivement indirectes, les récupérations réellement issues d'automatismes post-cablés. Au niveau théorique, le très faible score de reconnaissance montrerait que, pour la plupart des sujets, la marque n'a pas été directement encodée, en mémoire épisodique (mémoire des événements de vie), avec un indice qui rappellerait immédiatement la phase d'exposition au programme. En réexposant les sujets aux signes de marque, les questionnaires auraient dû, en cas d'encodage de la marque avec un indice, provoquer immédiatement le rappel de ce dernier (e.g. le programme ou un constituant du contexte socio-physicotemporel de l'expérience).

\section{- Des réactions affectives aux programmes réellement contrastées (tableaux 5 à 7 )}

En résumé, par rapport au programme gai, le programme triste est jugé comme étant davantage triste, tragique et attristant. Logiquement, ce programme est significativement moins gai, amusant et drôle que l'autre programme. Il a, en outre, réellement diminué les états affectifs «positifs » préalables des sujets de manière significative : ils sont moins joyeux, moins amusés, moins enthousiastes après le visionnage, moins enchantés, moins agréablement surpris et moins satisfaits pour GE. Il a également provoqué ou augmenté des affects "négatifs »: les sujets sont davantage tristes et dégoûtés.

Par rapport au programme triste, le programme gai est jugé, d'une part, comme étant plus gai, plus amusant, plus drôle et plus surprenant et, d'autre part, comme logiquement, moins triste, moins tragique et moins attristant. Il n'a pas obtenu de résultats aussi significatifs sur les états affectifs et l'intensité des émotions que le programme triste. Les résultats sont plus nets chez les étudiants en communication : ils sont plus amusés et moins tristes après l'exposition 17 (pour plus de détails, voir les tableaux 5 à 7).

17. Ces réactions n'ont pas été retrouvées chez les informaticiens où le programme gai aurait provoqué une certaine déception (accroissement de l'état « dégôuté »; ils sont moins enthousiastes). 
Tableau 5. - Evaluations affectives des programmes et tests de comparaison*

\begin{tabular}{|l|c|c|c|c|c|c|}
\hline \multirow{2}{*}{} & \multicolumn{2}{|c|}{$\begin{array}{c}\text { Moyennes } \\
\text { des }\end{array}$} & \multicolumn{3}{c|}{ Tests de comparaison } \\
\cline { 2 - 6 } & \multicolumn{2}{|c|}{ deux programmes } & \multicolumn{3}{c|}{ Comparaison des variances } & \multicolumn{2}{c|}{ Comparaison des moyennes } \\
\hline & $\begin{array}{c}\text { program. } \\
\text { triste GI } \\
n=34\end{array}$ & $\begin{array}{c}\text { Program. } \\
\text { gai GI } \\
n=33\end{array}$ & $\begin{array}{c}\text { Valeur } \\
\text { du } F\end{array}$ & Significativité & $\begin{array}{c}\text { Valeur } \\
\text { du } t\end{array}$ & $\begin{array}{c}\text { Significativité } \\
\text { (bilat.) }\end{array}$ \\
\hline Triste & 2,68 & 0,60 & 7,35 & $p<.01$ & $-6,69$ & $p<.001$ \\
Tragique & 2,44 & 0,85 & 11,18 & $p=.001$ & $-4,98$ & $p<.001$ \\
Décevant & 1,74 & 1,79 & 1,13 & $\mathrm{NS}$ & $-0,09$ & $\mathrm{NS}$ \\
Irritant & 1,09 & 0,67 & 1,33 & $\mathrm{NS}$ & $-1,31$ & $\mathrm{NS}$ \\
Ennuyeux & 2,09 & 1,45 & 1,28 & $\mathrm{NS}$ & $-1,58$ & $\mathrm{NS}$ \\
Donne le cafard & 0,74 & 0,48 & 0,77 & $\mathrm{NS}$ & $-0,94$ & $\mathrm{NS}$ \\
Attristant & 2,53 & 0,76 & 5,03 & $p<.05$ & $-5,34$ & $\mathrm{p}<.001$ \\
Gai & 0,12 & 1,48 & 44,42 & $p<.001$ & 5,86 & $\mathrm{p}<.001$ \\
Amusant & 0,71 & 2,54 & 0,88 & $\mathrm{NS}$ & 6,15 & $\mathrm{p}<.001$ \\
Drôle & 0,41 & 2,12 & 7,95 & $p<.01$ & 5,67 & $\mathrm{p}<.001$ \\
Surprenant & 1,62 & 3,03 & 2,77 & $\mathrm{NS}$ & 3,87 & $\mathrm{p}<.001$ \\
Excitant & 0,23 & 0,61 & 11,10 & $p=.001$ & 1,83 & $\mathrm{NS}(p=.07)$ \\
\hline
\end{tabular}

* Codage des réponses sur les échelles d'évaluation : 0 correspond à « pas du tout ", 5 à extrêmement.

Pour le codage des groupes, voir figure 1.

La marque Calmin est donc bien associée à deux programmes contenant des thèmes identiques mais provoquant réellement des réactions affectives soit négatives soit positives.

\section{Résultats concernant les effets automatiques des actions}

\section{- Résultats concernant les impressions de familiarité envers la marque (tableaux 8)}

Chez GI, l'action de parrainage du programme triste n'a pas eu davantage d'effet sur l'impression de familiarité comparé aux sujets non exposés. Les résultats sont différents pour le programme gai : son parrainage a eu un réel effet. La marque est considérée comme familière par plus de sujets (pour Existe : $\chi^{2}=14,62, p<.001 ; \chi^{2}=9,36, p<.01$ pour Contact et $\chi^{2}=6,02, p=.01$ pour Copidess). Cet effet est constaté en comparant avec les sujets non exposés (chez GE : Existe : $\chi^{2}=5,98, p<.001$; Contact : $\left.\chi^{2}=7,55, p<.01\right)$ mais, plus surprenant, avec les sujets exposés au parrainage du programme triste (chez GI : Existe : $\chi^{2}=6,07, p<.01$; Copinom : $\left.\chi^{2}=4,51, p<.05\right)$. Les hypothèses $\mathrm{H} 1$ et $\mathrm{H} 2$ concernant les effets des programmes sur l'impression de familiarité sont invalidées : la différence dans les valeurs affectives des programmes a un effet sur l'impression de familiarité.

Si on devait retenir un unique item pour évaluer la familiarité, ce serait Existe : dans les trois tableaux où des résultats sont significatifs, il met à chaque fois en évidence la familiarité. Ses trois scores $C^{18}$ sont, en effet, les plus élevés : même s'ils n'ont aucune signification statistique, ils donnent une certaine idée de la «pertinence opérationnelle » de la variable. Il n'est cependant pas possible d'indiquer si c'est le libellé de l'item ou sa première position parmi les quatre questions qui lui confère cette sensibilité.

- Résultats concernant le modèle de mésattribution de la familiarité ( I $^{\text {re }}$ section du tableau 9)

La première étape du modèle, indépendante de cette seconde étape, a été invalidée. N'observant pas

18. $C$ est ici le coefficient de contingence : il estime la corrélation ou, plus exactement, la force de l'association entre deux variables qualitatives. 
Tableau 8. - Résultats concernant les items enregistrant l'impression de familiarité

\begin{tabular}{|c|c|c|c|c|c|}
\hline & & $\begin{array}{c}\text { GI triste } \\
n=30\end{array}$ & $\begin{array}{c}\text { GCI } \\
n=29\end{array}$ & $\begin{array}{c}\chi^{2} \\
\text { (*correction } \\
\text { de Yates) }\end{array}$ & $\mathrm{C}$ \\
\hline \multirow{2}{*}{ Existe } & oui & $\begin{array}{c}8 \\
(5,6)\end{array}$ & $\begin{array}{c}3 \\
(5,4)\end{array}$ & \multirow[t]{2}{*}{$1,63^{*}$} & \multirow[t]{2}{*}{0,20} \\
\hline & non & $\begin{array}{c}22 \\
(24,4)\end{array}$ & $\begin{array}{c}26 \\
(23,6)\end{array}$ & & \\
\hline \multirow{2}{*}{ Contact } & oui & $\begin{array}{c}10 \\
(7,1)\end{array}$ & $\begin{array}{c}4 \\
(16,9)\end{array}$ & \multirow[t]{2}{*}{$2,12 *$} & \multirow[t]{2}{*}{0,22} \\
\hline & non & $\begin{array}{c}20 \\
(22,9)\end{array}$ & $\begin{array}{c}25 \\
(22,1)\end{array}$ & & \\
\hline \multirow{2}{*}{ Copinom } & oui & $\begin{array}{c}14 \\
(14,7)\end{array}$ & $\begin{array}{c}15 \\
(14,3)\end{array}$ & \multirow[t]{2}{*}{0,15} & \multirow[t]{2}{*}{0,05} \\
\hline & non & $\begin{array}{c}16 \\
(15,3)\end{array}$ & $\begin{array}{c}14 \\
(14,7)\end{array}$ & & \\
\hline \multirow{2}{*}{ Copidess } & oui & $\begin{array}{c}13 \\
(9,7)\end{array}$ & $\begin{array}{c}6 \\
(9,3)\end{array}$ & \multirow[t]{2}{*}{$2,50 *$} & \multirow[t]{2}{*}{0,24} \\
\hline & non & $\begin{array}{c}17 \\
(20,3)\end{array}$ & $\begin{array}{c}23 \\
(19,7)\end{array}$ & & \\
\hline
\end{tabular}

\begin{tabular}{|c|c|c|c|c|c|}
\hline & $\begin{array}{l}\text { GI gai } \\
n=31\end{array}$ & $\begin{array}{l}\text { GI triste } \\
n=30\end{array}$ & $\begin{array}{c}\chi^{2} \\
\text { (*correction } \\
\text { de Yates) }\end{array}$ & $\mathrm{C}$ \\
\hline \multirow{2}{*}{ Existe } & oui & $\begin{array}{c}19 \\
(13,7)\end{array}$ & $\begin{array}{c}8 \\
(13,3)\end{array}$ & \multirow[t]{2}{*}{$6,07 * c$} & \multirow[t]{2}{*}{0,33} \\
\hline & non & $\begin{array}{c}12 \\
(17,3)\end{array}$ & $\begin{array}{c}22 \\
(16,7)\end{array}$ & & \\
\hline \multirow{2}{*}{ Contact } & oui & $\begin{array}{c}17 \\
(13,7)\end{array}$ & $\begin{array}{c}10 \\
(13,3)\end{array}$ & \multirow[t]{2}{*}{2,85} & \multirow[t]{2}{*}{0,21} \\
\hline & non & $\begin{array}{c}14 \\
(17,3)\end{array}$ & $\begin{array}{c}20 \\
(16,7)\end{array}$ & & \\
\hline \multirow{2}{*}{ Copinom } & oui & $\begin{array}{c}23 \\
(18,5)\end{array}$ & $\begin{array}{c}14 \\
(11,5)\end{array}$ & \multirow[t]{2}{*}{$4,51 * \mathrm{c}$} & \multirow[t]{2}{*}{0,29} \\
\hline & non & $\begin{array}{c}7 \\
(11,5)\end{array}$ & $\begin{array}{c}16 \\
(11,5)\end{array}$ & & \\
\hline \multirow{2}{*}{ Copidess } & oui & $\begin{array}{c}17 \\
(15,2)\end{array}$ & $\begin{array}{c}13 \\
(14,8)\end{array}$ & \multirow[t]{2}{*}{0,81} & \multirow[t]{2}{*}{0,11} \\
\hline & non & $\begin{array}{c}14 \\
(15,8)\end{array}$ & $\begin{array}{c}17 \\
(15,2)\end{array}$ & & \\
\hline
\end{tabular}

Tableau croisé et test du $\chi^{2}$ pour GI : programme triste - GC.

Tableau croisé et test du $\chi^{2}$ pour GI : programme gai - triste.

\begin{tabular}{|c|c|c|c|c|c|}
\hline & & $\begin{array}{l}\text { GI gai } \\
n=30\end{array}$ & $\begin{array}{c}\mathrm{GCI} \\
n=29\end{array}$ & $\begin{array}{c}\chi^{2} \\
\text { (*correction } \\
\text { de Yates) }\end{array}$ & C \\
\hline & oui & $\begin{array}{c}19 \\
(11,4)\end{array}$ & $\begin{array}{c}3 \\
(10,6)\end{array}$ & $14,62 * a$ & 0,47 \\
\hline Existe & non & $\begin{array}{c}12 \\
(19,6)\end{array}$ & $\begin{array}{c}26 \\
(18,4)\end{array}$ & & \\
\hline & oui & $\begin{array}{c}17 \\
(10,9)\end{array}$ & $\begin{array}{c}4 \\
(10,2)\end{array}$ & $9,36 * \mathrm{~b}$ & 0,39 \\
\hline Comtact & non & $\begin{array}{c}14 \\
(20,2)\end{array}$ & $\begin{array}{c}25 \\
(18,9)\end{array}$ & & \\
\hline & oui & $\begin{array}{c}23 \\
(19,3)\end{array}$ & $\begin{array}{c}15 \\
(18,7)\end{array}$ & $2,99 *$ & 0,25 \\
\hline Copinom & non & $\begin{array}{c}7 \\
(10,7)\end{array}$ & $\begin{array}{c}14 \\
(10,3)\end{array}$ & & \\
\hline & oui & $\begin{array}{c}17 \\
(11,9)\end{array}$ & $\begin{array}{c}6 \\
(11,1)\end{array}$ & $6,02 * c$ & 0,33 \\
\hline & non & $\begin{array}{c}14 \\
(19,1)\end{array}$ & $\begin{array}{c}23 \\
(17,9)\end{array}$ & & \\
\hline
\end{tabular}

\begin{tabular}{|c|c|c|c|c|c|}
\hline & & $\begin{array}{l}\text { GE gai } \\
n=19\end{array}$ & $\begin{array}{c}\text { GCE } \\
n=27\end{array}$ & $\begin{array}{c}\chi^{2} \\
\text { (*correction } \\
\text { de Yates) }\end{array}$ & $\mathrm{C}$ \\
\hline \multirow{2}{*}{ Existe } & oui & $\begin{array}{c}8 \\
(4,1)\end{array}$ & $\begin{array}{c}2 \\
(5,9)\end{array}$ & \multirow[t]{2}{*}{$5,98 * \mathrm{c}$} & \multirow[t]{2}{*}{0,38} \\
\hline & non & $\begin{array}{c}11 \\
(14,9)\end{array}$ & $\begin{array}{c}25 \\
(21,1)\end{array}$ & & \\
\hline \multirow{2}{*}{ Contact } & oui & $\begin{array}{c}10 \\
(5,4)\end{array}$ & $\begin{array}{c}3 \\
(7,6)\end{array}$ & \multirow[t]{2}{*}{$7,55^{* b}$} & \multirow[t]{2}{*}{0,41} \\
\hline & non & $\begin{array}{c}9 \\
(13,6)\end{array}$ & $\begin{array}{c}24 \\
(19,4)\end{array}$ & & \\
\hline \multirow{2}{*}{ Copinom } & oui & $\begin{array}{c}11 \\
(8,7)\end{array}$ & $\begin{array}{c}10 \\
(12,3)\end{array}$ & \multirow[t]{2}{*}{$1,21 *$} & \multirow[t]{2}{*}{0,20} \\
\hline & non & $\begin{array}{c}8 \\
(10,3)\end{array}$ & $\begin{array}{c}17 \\
(14,7)\end{array}$ & & \\
\hline \multirow{2}{*}{ Copidess } & oui & $\begin{array}{c}6 \\
(39,7)\end{array}$ & $\begin{array}{c}3 \\
(5,3)\end{array}$ & \multirow[t]{2}{*}{$1,81 *$} & \multirow[t]{2}{*}{0,25} \\
\hline & non & $\begin{array}{c}13 \\
(15,3)\end{array}$ & $\begin{array}{c}24 \\
(21,7)\end{array}$ & & \\
\hline
\end{tabular}

Tableau croisé et test du $\chi^{2}$ pour GI : programme gai - GC.

Tableau croisé et test du $\chi^{2}$ pour GE : programme gai - GC.

Note : la significativité est représentée par ${ }^{\mathrm{a}} p<.001 ;{ }^{\mathrm{b}} p<.01 ;{ }^{\mathrm{c}} p<.05$. 
Tableau 9. - Résultats concernant les items d'évaluation affective et les attributs marketing (1 ${ }^{\text {re }}$ section) et les items enregistrant le transfert sémantique automatique $\left(2^{\mathrm{e}}\right.$ section)

\begin{tabular}{|c|c|c|c|c|c|c|c|c|c|c|c|c|c|c|c|c|c|}
\hline \multirow[b]{2}{*}{ VD } & \multirow[b]{2}{*}{ Sou } & \multicolumn{4}{|c|}{ GI : prog. triste-GCI } & \multicolumn{4}{|c|}{ GI : prog. gai -GCI } & \multicolumn{4}{|c|}{ GI : prog. gai-triste } & \multicolumn{4}{|c|}{ GE : gai-GCE } \\
\hline & & ddl & SC & $\mathbf{C M}$ & $\mathbf{F}$ & ddl & SC & CM & $\mathbf{F}$ & ddl & $\mathrm{SC}$ & $\mathbf{C M}$ & F & ddl & SC & CM & F \\
\hline Plaisant & $\begin{array}{l}\mathrm{Vi} \\
\text { Err } \\
\text { tot }\end{array}$ & $\begin{array}{r}1 \\
57 \\
58\end{array}$ & $\begin{array}{r}, 34 \\
42,29 \\
42,64\end{array}$ & $\begin{array}{l}, 34 \\
, 74\end{array}$ & ,47 & $\begin{array}{r}1 \\
58 \\
59\end{array}$ & $\begin{array}{r}, 08 \\
50,50 \\
50,58\end{array}$ & $\begin{array}{l}, 07 \\
, 88\end{array}$ &, 09 & $\begin{array}{r}1 \\
\frac{59}{60}\end{array}$ & $\begin{array}{r}, 101 \\
\frac{43,14}{43,24}\end{array}$ & $\begin{array}{l}, 10 \\
, 73\end{array}$ & ,14 & $\begin{array}{r}1 \\
\frac{41}{42}\end{array}$ & $\begin{array}{r}1,06 \\
\frac{35,68}{36,74}\end{array}$ & $\begin{array}{r}1,06 \\
, 87\end{array}$ & 1,22 \\
\hline Choix & $\begin{array}{l}\mathrm{Vi} \\
\text { Err } \\
\text { tot }\end{array}$ & $\begin{array}{r}1 \\
57 \\
58\end{array}$ & $\begin{array}{r}, 17 \\
61,0 \\
61,18\end{array}$ & $\begin{array}{r}, 17 \\
1,07\end{array}$ &, 16 & $\begin{array}{r}1 \\
58 \\
59\end{array}$ & $\begin{array}{r}, 4 \\
\frac{69.95}{70,40}\end{array}$ & $\begin{array}{r}45 \\
1,20\end{array}$ & ,37 & $\begin{array}{r}1 \\
59 \\
60\end{array}$ & $\begin{array}{r}1,24 \\
70,54 \\
71,77\end{array}$ & $\begin{array}{l}1,23 \\
1,19\end{array}$ & 1,03 & $\begin{array}{r}1 \\
\frac{41}{42}\end{array}$ & $\begin{array}{r}4,30 \\
33,88 \\
38,19\end{array}$ & $\begin{array}{r}4,30 \\
, 83\end{array}$ & $5,21^{\mathrm{c}}$ \\
\hline Imagprod & $\begin{array}{l}\mathrm{Vi} \\
\underline{\text { Err }} \\
\text { tot }\end{array}$ & $\begin{array}{r}1 \\
57 \\
58\end{array}$ & $\begin{array}{r}, 94 \\
\frac{35,70}{36,64}\end{array}$ & $\begin{array}{l}, 94 \\
, 62\end{array}$ & 1,50 & $\begin{array}{r}1 \\
58 \\
59\end{array}$ & $\begin{array}{r}, 15 \\
44.77 \\
44,93\end{array}$ & $\begin{array}{l}, 15 \\
, 77\end{array}$ & ,20 & $\begin{array}{r}1 \\
\frac{59}{60}\end{array}$ & $\begin{array}{r}, 34 \\
\frac{38.41}{38,75}\end{array}$ & $\begin{array}{l}, 34 \\
, 65\end{array}$ &, 53 & $\begin{array}{r}1 \\
\frac{44}{45}\end{array}$ & $\begin{array}{r}, 12 \\
37.03 \\
37,15\end{array}$ & $\begin{array}{l}, 12 \\
, 84\end{array}$ &, 14 \\
\hline Gamme & $\begin{array}{l}\mathrm{Vi} \\
\frac{\mathrm{Err}}{\text { tot }}\end{array}$ & $\begin{array}{r}1 \\
57 \\
58\end{array}$ & $\begin{array}{r}, 00 \\
\underline{59,93} \\
59,93\end{array}$ & $\begin{array}{r}, 00 \\
1,05\end{array}$ &, 00 & $\begin{array}{r}1 \\
58 \\
59\end{array}$ & $\begin{array}{r}3,08 \\
\underline{58.51} \\
61,60\end{array}$ & $\begin{array}{l}3,08 \\
1,01\end{array}$ & $3,06^{\mathbf{c}}$ & $\begin{array}{r}1 \\
\underline{59} \\
60\end{array}$ & $\begin{array}{r}3,12 \\
\frac{56,51}{59,64}\end{array}$ & $\begin{array}{r}3,12 \\
, 96\end{array}$ & $3,26^{\mathrm{c}}$ & $\begin{array}{r}1 \\
44 \\
45\end{array}$ & $\begin{array}{r}2,97 \\
\frac{41.83}{44,80}\end{array}$ & $\begin{array}{r}2,97 \\
, 95\end{array}$ & $3,13^{\mathrm{c}}$ \\
\hline Ageinven & $\begin{array}{l}\mathrm{Vi} \\
\mathrm{Err} \\
\text { tot }\end{array}$ & $\begin{array}{r}1 \\
57 \\
58\end{array}$ & $\begin{array}{r}1,31 \\
\frac{61.63}{62,94}\end{array}$ & $\begin{array}{l}1,31 \\
1,08\end{array}$ & 1,21 & $\begin{array}{r}1 \\
58 \\
59\end{array}$ & $\begin{array}{r}, 07 \\
77,93 \\
78,00\end{array}$ & $\begin{array}{r}, 06 \\
1,34\end{array}$ & 05 & $\begin{array}{r}1 \\
\frac{59}{60}\end{array}$ & $\begin{array}{r}2,04 \\
\underline{53,63} \\
55,67\end{array}$ & $\begin{array}{r}2,04 \\
, 91\end{array}$ & 2,24 & $\begin{array}{r}1 \\
\frac{44}{45}\end{array}$ & $\begin{array}{r}73 \\
\underline{34,42} \\
35,15\end{array}$ & $\begin{array}{l}, 73 \\
, 78\end{array}$ &, 93 \\
\hline
\end{tabular}

\begin{tabular}{|c|c|c|c|c|c|c|c|c|c|c|c|c|c|c|c|c|c|}
\hline Femme & $\begin{array}{l}\mathrm{Vi} \\
\text { Err } \\
\text { tot }\end{array}$ & $\begin{array}{r}1 \\
57 \\
58\end{array}$ & $\begin{array}{r}, 05 \\
90,62 \\
90,67\end{array}$ & $\begin{array}{r}, 05 \\
1,58\end{array}$ & ,03 & $\begin{array}{r}1 \\
58 \\
59\end{array}$ & $\begin{array}{r}3,31 \\
93,53 \\
96,85\end{array}$ & $\begin{array}{l}3,32 \\
1,61\end{array}$ & 2,06 & $\begin{array}{r}1 \\
59 \\
60\end{array}$ & $\begin{array}{r}2,57 \\
78,64 \\
81,21\end{array}$ & $\begin{array}{l}2,57 \\
1,33\end{array}$ & 1,93 & $\begin{array}{r}1 \\
44 \\
45\end{array}$ & $\begin{array}{r}, 00 \\
\frac{59.15}{59,15}\end{array}$ & $\begin{array}{l}, 002 \\
1,34\end{array}$ & ,00 \\
\hline Homme & $\begin{array}{l}\mathrm{Vi} \\
\text { Err } \\
\text { tot }\end{array}$ & $\begin{array}{r}1 \\
57 \\
58\end{array}$ & $\left\{\begin{array}{r}, 07 \\
\frac{64,83}{64,91}\end{array}\right.$ & $\begin{array}{r}, 07 \\
1,13\end{array}$ & ,06 & $\begin{array}{r}1 \\
58 \\
59\end{array}$ & $\begin{array}{r}, 00 \\
\frac{80.33}{80,33}\end{array}$ & $\begin{array}{r}, 00 \\
1,38\end{array}$ & ,00 & $\begin{array}{r}1 \\
59 \\
60\end{array}$ & $\begin{array}{r}, 06 \\
60.89 \\
98,75\end{array}$ & $\begin{array}{r}06 \\
1,03\end{array}$ & ,05 & $\begin{array}{r}1 \\
\frac{44}{45}\end{array}$ & $\begin{array}{r}2,73 \\
50,48 \\
53,22\end{array}$ & $\begin{array}{l}2,73 \\
1,15\end{array}$ & 2,38 \\
\hline-20 ans & $\begin{array}{l}\mathrm{Vi} \\
\text { Err } \\
\text { tot }\end{array}$ & $\begin{array}{r}1 \\
57 \\
58\end{array}$ & $\begin{array}{r}, 32 \\
\frac{101,47}{101,79}\end{array}$ & $\begin{array}{r}, 32 \\
1,78\end{array}$ & 18 & $\begin{array}{r}1 \\
58 \\
59\end{array}$ & $\begin{array}{r}, 07 \\
\frac{94,91}{94,98}\end{array}$ & $\begin{array}{l}069 \\
1,64\end{array}$ & ,04 & $\begin{array}{r}1 \\
59 \\
60\end{array}$ & $\begin{array}{r}, 71 \\
98,04 \\
98,75\end{array}$ & $\begin{array}{r}, 71 \\
1,66\end{array}$ & ,43 & $\begin{array}{r}1 \\
44 \\
45\end{array}$ & $\begin{array}{r}2,55 \\
74,08 \\
76,61\end{array}$ & $\begin{array}{l}2,52 \\
1,68\end{array}$ & 1,49 \\
\hline $\begin{array}{l}\text { entre } 20 \\
\text { et } 40 \text { ans }\end{array}$ & $\begin{array}{l}\mathrm{Vi} \\
\text { Err } \\
\text { tot }\end{array}$ & $\begin{array}{r}1 \\
57 \\
58\end{array}$ & $\begin{array}{r}, 39 \\
79,22 \\
79,62\end{array}$ & $\begin{array}{r}, 39 \\
1,39\end{array}$ & ,28 & $\begin{array}{r}1 \\
58 \\
59\end{array}$ & $\begin{array}{r}, 00 \\
\frac{87,73}{87,73}\end{array}$ & $\begin{array}{l}, 000 \\
1,51\end{array}$ & ,00 & $\begin{array}{r}1 \\
\underline{59} \\
60\end{array}$ & $\begin{array}{r}, 43 \\
83,24 \\
83,67\end{array}$ & $\begin{array}{r}, 43 \\
1,41\end{array}$ &, 31 & $\begin{array}{r}1 \\
44 \\
45\end{array}$ & $\begin{array}{r}, 03 \\
\frac{50,40}{50,43}\end{array}$ & $\begin{array}{r}, 03 \\
1,14\end{array}$ & ,03 \\
\hline $\begin{array}{l}\text { entre } 40 \\
\text { et } 60 \text { ans }\end{array}$ & $\begin{array}{l}\mathrm{Vi} \\
\text { Err } \\
\text { tot }\end{array}$ & $\begin{array}{r}1 \\
57 \\
58\end{array}$ & $\begin{array}{r}, 92 \\
\frac{67,10}{68,03}\end{array}$ & $\begin{array}{r}, 92 \\
1,17\end{array}$ & ,78 & $\begin{array}{r}1 \\
57 \\
58\end{array}$ & $\begin{array}{r}, 060 \\
\frac{64,79}{64,85}\end{array}$ & $\begin{array}{l}060 \\
1,11\end{array}$ & ,053 & $\begin{array}{r}1 \\
59 \\
60\end{array}$ & $\begin{array}{r}1,50 \\
57,41 \\
58,92\end{array}$ & $\begin{array}{r}1,50 \\
, 97\end{array}$ & 1,54 & $\begin{array}{r}1 \\
\frac{44}{45}\end{array}$ & $\begin{array}{r}, 11 \\
55,82 \\
55,93\end{array}$ & $\begin{array}{r}11 \\
1,27\end{array}$ & ,09 \\
\hline $\begin{array}{l}\text { Supérieur } \\
\text { à } 60 \text { ans }\end{array}$ & $\begin{array}{l}\mathrm{Vi} \\
\text { Err } \\
\text { tot }\end{array}$ & $\begin{array}{r}1 \\
56 \\
57\end{array}$ & $\begin{array}{r}, 27 \\
\frac{113,72}{114,00}\end{array}$ & $\begin{array}{r}, 27 \\
2,03\end{array}$ & 13 & $\begin{array}{r}1 \\
57 \\
58\end{array}$ & $\begin{array}{r}, 35 \\
\frac{113,27}{113,62}\end{array}$ & $\begin{array}{l}350 \\
1,99\end{array}$ & , 18 & $\begin{array}{r}1 \\
59 \\
60\end{array}$ & $\begin{array}{r}1,30 \\
115,29 \\
116,59\end{array}$ & $\begin{array}{l}1,30 \\
1,95\end{array}$ & ,67 & $\begin{array}{r}1 \\
44 \\
45\end{array}$ & $\begin{array}{r}, 00 \\
59.48 \\
59,48\end{array}$ & $\begin{array}{r}, 00 \\
1,35\end{array}$ & ,00 \\
\hline Affectci & $\begin{array}{l}\mathrm{Vi} \\
\text { Err } \\
\text { tot }\end{array}$ & $\begin{array}{r}1 \\
57 \\
58\end{array}$ & $\begin{array}{r}, 00 \\
59,1 \\
59,1\end{array}$ & $\begin{array}{r}, 00 \\
1,34\end{array}$ & ,09 & $\begin{array}{r}1 \\
57 \\
58\end{array}$ & $\begin{array}{r}, 36 \\
91.89 \\
92,26\end{array}$ & $\begin{array}{r}, 36 \\
1,56\end{array}$ & ,23 & $\begin{array}{r}1 \\
59 \\
60\end{array}$ & $\begin{array}{r}, 44 \\
47,78 \\
48,23\end{array}$ & $\begin{array}{l}, 44 \\
, 81\end{array}$ &, 55 & $\begin{array}{r}1 \\
44 \\
45\end{array}$ & $\begin{array}{r}, 00 \\
59.94 \\
59,95\end{array}$ & $\begin{array}{r}, 00 \\
1,05\end{array}$ & ,00 \\
\hline Ocasgai & $\begin{array}{l}\mathrm{Vi} \\
\text { Err } \\
\text { tot }\end{array}$ & $\begin{array}{r}1 \\
57 \\
58\end{array}$ & $\begin{array}{r}2,44 \\
75,55 \\
78,00\end{array}$ & $\begin{array}{l}2,44 \\
1,32\end{array}$ & 1,84 & $\begin{array}{r}1 \\
57 \\
58\end{array}$ & $\begin{array}{r}4,72 \\
77,85 \\
82,58\end{array}$ & $\begin{array}{l}4,73 \\
1,34\end{array}$ & $3,52^{\mathrm{c}}$ & $\begin{array}{r}1 \\
\underline{59} \\
60\end{array}$ & $\begin{array}{r}, 36 \\
91,90 \\
92,26\end{array}$ & $\begin{array}{r}, 36 \\
1,56\end{array}$ &, 23 & $\begin{array}{r}1 \\
44 \\
45\end{array}$ & $\begin{array}{r}19 \\
55,02 \\
55,22\end{array}$ & $\begin{array}{r}19 \\
1,25\end{array}$ & 16 \\
\hline Ocastri & $\begin{array}{l}\mathrm{Vi} \\
\text { Err } \\
\text { tot }\end{array}$ & $\begin{array}{r}1 \\
57 \\
58\end{array}$ & $\begin{array}{r}2,25 \\
\frac{78.45}{80,71}\end{array}$ & $\begin{array}{l}2,25 \\
1,36\end{array}$ & 1,63 & $\begin{array}{r}1 \\
58 \\
59\end{array}$ & $\begin{array}{l}10,10 \\
85,50 \\
95,60\end{array}$ & $\begin{array}{l}10,1 \\
1,49\end{array}$ & $6,77^{b}$ & $\begin{array}{r}1 \\
59 \\
60\end{array}$ & $\begin{array}{r}2,82 \\
101,38 \\
104,20\end{array}$ & $\begin{array}{l}2,82 \\
1,72\end{array}$ & 1,64 & $\begin{array}{r}1 \\
44 \\
45\end{array}$ & $\begin{array}{r}1,14 \\
\frac{63,82}{64,96}\end{array}$ & $\begin{array}{l}1,14 \\
1,45\end{array}$ & ,78 \\
\hline
\end{tabular}


Tableau 9. - (Suite)

\begin{tabular}{|c|c|c|c|c|c|c|c|c|c|c|c|c|c|c|c|c|c|}
\hline \multirow[b]{2}{*}{ VD } & \multirow[b]{2}{*}{ Sou } & \multicolumn{4}{|c|}{ GI : prog. triste-GCI } & \multicolumn{4}{|c|}{ GI : prog. gai-GCI } & \multicolumn{4}{|c|}{ GI : prog. gai-triste } & \multicolumn{4}{|c|}{ GE : gai-GCE } \\
\hline & & ddl & SC & CM & F & ddl & SC & $\mathrm{CM}$ & F & ddl & SC & $\mathrm{CM}$ & $\mathbf{F}$ & dd & SC & CM & F \\
\hline Cotillon & $\begin{array}{l}\mathrm{Vi} \\
\text { Err } \\
\text { tot }\end{array}$ & $\begin{array}{r}1 \\
57 \\
58\end{array}$ & $\begin{array}{r}1,01 \\
\frac{130,64}{131,66}\end{array}$ & $\begin{array}{l}1,01 \\
2,29\end{array}$ & ,44 & $\begin{array}{r}1 \\
58 \\
59\end{array}$ & $\begin{array}{r}, 70 \\
1 \underline{34,55} \\
135,25\end{array}$ & $\begin{array}{r}, 70 \\
2,32\end{array}$ &, 30 & $\begin{array}{r}1 \\
\underline{59} \\
60\end{array}$ & $\begin{array}{r}, 03 \\
134,30 \\
134,33\end{array}$ & $\begin{array}{r}, 03 \\
2,28\end{array}$ & 01 & $\frac{4}{4}$ & $\begin{array}{r}2,6 \\
114 \\
116\end{array}$ & $\begin{array}{l}2,63 \\
2,60\end{array}$ & 1,01 \\
\hline Jouets & $\begin{array}{l}\mathrm{Vi} \\
\text { Err } \\
\text { tot }\end{array}$ & $\begin{array}{r}1 \\
57 \\
58\end{array}$ & $\begin{array}{r}5,08 \\
\frac{129,79}{134,88}\end{array}$ & $\begin{array}{l}5,08 \\
2,27\end{array}$ & 2,23 & $\begin{array}{r}1 \\
58 \\
59\end{array}$ & $\begin{array}{l}11,57 \\
\frac{118,76}{130,33}\end{array}$ & $\begin{array}{l}11,6 \\
2,05\end{array}$ & $5,65^{\mathrm{c}}$ & $\begin{array}{r}1 \\
\frac{59}{60}\end{array}$ & $\begin{array}{r}1,29 \\
126.90 \\
128,20\end{array}$ & $\begin{array}{l}1,29 \\
2,15\end{array}$ & 60 & $\frac{4}{4}$ & $\begin{array}{r}2,4 \\
113 \\
116\end{array}$ & $\begin{array}{l}2,42 \\
2,58\end{array}$ &, 94 \\
\hline Detresfe & $\begin{array}{l}\mathrm{Vi} \\
\text { Err } \\
\text { tot }\end{array}$ & $\begin{array}{r}1 \\
57 \\
58\end{array}$ & $\begin{array}{r}, 11 \\
\frac{122,29}{122,40}\end{array}$ & $\begin{array}{r}, 11 \\
2,14\end{array}$ & ,05 & $\begin{array}{r}1 \\
58 \\
59\end{array}$ & $\begin{array}{r}2,15 \\
\frac{142,83}{144,98}\end{array}$ & $\begin{array}{l}2,15 \\
2,46\end{array}$ &, 87 & $\begin{array}{r}1 \\
\frac{59}{60}\end{array}$ & $\begin{array}{r}3,32 \\
127,47 \\
130,79\end{array}$ & $\begin{array}{l}3,32 \\
2,16\end{array}$ &, 15 & $\frac{4}{4}$ & $\begin{array}{r}, 3 \\
84,5 \\
84,8\end{array}$ & $\begin{array}{r}, 34 \\
1,92\end{array}$ & ,18 \\
\hline Funerair & $\begin{array}{l}\mathrm{Vi} \\
\text { Err } \\
\text { tot }\end{array}$ & $\begin{array}{r}1 \\
57 \\
58\end{array}$ & $\begin{array}{r}, 84 \\
\underline{98,00} \\
98,84\end{array}$ & $\begin{array}{r}, 84 \\
1,71\end{array}$ & ,49 & $\begin{array}{r}1 \\
58 \\
59\end{array}$ & $\begin{array}{r}, 84 \\
\underline{98.01} \\
98,85\end{array}$ & $\begin{array}{l}, 841 \\
1,68\end{array}$ &, 500 & $\begin{array}{r}1 \\
59 \\
60\end{array}$ & $\begin{array}{r}, 00 \\
\underline{122.74} \\
123,74\end{array}$ & $\begin{array}{r}, 00 \\
2,10\end{array}$ & 00 & 4 & $\frac{39 .}{39,}$ & $\begin{array}{l}, 05 \\
, 90\end{array}$ & ,06 \\
\hline Video 60 & $\begin{array}{l}\mathrm{Vi} \\
\text { Err } \\
\text { tot }\end{array}$ & $\begin{array}{r}1 \\
57 \\
58\end{array}$ & $\begin{array}{r}, 19 \\
\frac{81,83}{82,03}\end{array}$ & $\begin{array}{r}, 19 \\
1,43\end{array}$ &, 13 & $\begin{array}{r}1 \\
58 \\
59\end{array}$ & $\begin{array}{r}0,019 \\
\frac{100,91}{100,93}\end{array}$ & $\begin{array}{l}, 019 \\
1,73\end{array}$ & 010 & $\begin{array}{r}1 \\
59 \\
60\end{array}$ & $\begin{array}{r}, 34 \\
112,41 \\
112,75\end{array}$ & $\begin{array}{r}, 34 \\
1,90\end{array}$ & , 18 & 4 & $\begin{array}{r}1, \\
68 \\
70,\end{array}$ & $\begin{array}{l}1,78 \\
1,56\end{array}$ & 1,14 \\
\hline Scolaire & $\begin{array}{l}\mathrm{Vi} \\
\text { Err } \\
\text { tot }\end{array}$ & $\begin{array}{r}1 \\
57 \\
58\end{array}$ & $\begin{array}{r}, 17 \\
\frac{61,00}{61,18}\end{array}$ & $\begin{array}{r}17 \\
1,07\end{array}$ &, 16 & $\begin{array}{r}1 \\
58 \\
59\end{array}$ & $\begin{array}{r}\quad, 45 \\
69.95 \\
70,40\end{array}$ & $\begin{array}{l}, 451 \\
1,21\end{array}$ &, 37 & $\begin{array}{r}1 \\
59 \\
60\end{array}$ & $\begin{array}{r}, 18 \\
116,09 \\
116,26\end{array}$ & $\begin{array}{r}18 \\
1,97\end{array}$ &, 09 & & $\frac{59}{59}$ & $\begin{array}{r}, 00 \\
1,34\end{array}$ &, 00 \\
\hline TV HI-FI & $\begin{array}{l}V i \\
\text { Err } \\
\text { tot }\end{array}$ & $\begin{array}{r}1 \\
57 \\
58\end{array}$ & $\begin{array}{r}3,50 \\
90,29 \\
93,79\end{array}$ & $\begin{array}{l}3,50 \\
1,58\end{array}$ & 2,21 & $\begin{array}{r}1 \\
58 \\
59\end{array}$ & $\begin{array}{l}\frac{3,162}{97.02} \\
100,18\end{array}$ & $\begin{array}{l}3,16 \\
1,67\end{array}$ & 1,89 & $\begin{array}{r}1 \\
\frac{59}{60}\end{array}$ & $\begin{array}{r}, 01 \\
\frac{93.66}{93,67}\end{array}$ & $\begin{array}{l}, 011 \\
1,59\end{array}$ & 0 & 4 & $\begin{array}{c}4, \\
74 . \\
78,\end{array}$ & $\begin{array}{l}4,44 \\
1,69\end{array}$ & 2,63 \\
\hline
\end{tabular}

La significativité unilatérale est représentée par ${ }^{\mathrm{a}} p<.001 ;{ }^{\mathrm{b}} p<.01 ;^{\mathrm{c}} p<.05$.

d'effet de l'action de parrainage du programme triste sur l'impression de familiarité, nous ne devrions pas constater d'effet sur les variables d'évaluation affective et les attributs marketing. Comme le postule $\mathrm{H} 3 \mathrm{~b}$, c'est effectivement le cas : aucune différence entre le groupe exposé au programme triste (GI) et le groupe contrôle n'est enregistrée. Le parrainage du programme gai a eu, quant à lui, des effets sur les variables d'évaluation affective et sur les attributs marketing. Ces effets sont différents selon les groupes :

- L'action a eu des effets positifs sur les évaluations affectives de la marque, jugée mieux choisie chez GE $(2,35$ contre 3 pour GCE ; $F=5,21, p<.05)$. Elle n'a pas eu d'effet sur ces évaluations affectives chez GI.

- L'action a eu des effets positifs sur la hauteur de la gamme de la marque dans les deux types d'échantillons : chez GI, la hauteur de gamme des produits est perçue comme étant plus élevée $(2,58$ contre 3,03 pour le programme triste ; $F=3,26, p<.05 ; 2,58$ contre 3,03 pour $\mathrm{GCI} ; \mathrm{F}=3,06, p<.05)$, les résultats sont identiques chez GE (2,63 contre 3,15 pour GCE ; $F=3,13, p<.05$ ).

Ces derniers résultats vont dans le sens des hypothèses H3 (H3b est validée) ${ }^{19}$. Il va s'agir cependant de revoir la première étape du modèle.

\section{- Résultats concernant l'absence de transfert sémantique automatique ( $2^{e}$ section du tableau 9)}

On n'enregistre aucune différence sur les variables marketing transférées de la thématique des pro-

19. En suivant la logique épistémologique de Popper, il est plus convenable de conclure que cette expérimentation apporte des preuves « en faveur » du modèle. 
grammes, du contexte et du canal entre les groupes exposés aux programmes et les non-exposés ainsi qu'entre les exposés aux programmes triste et gai. L'hypothèse de l'absence de transfert sémantique automatique (H4) est validée.

Les résultats mettent cependant en évidence une influence de l'action de parrainage du programme gai, d'une part, sur les occasions de consommation : la marque est plus adaptée aux occasions tristes de consommation ( 3,10 contre 2,28 pour le $\mathrm{GC} ; F=6,77$; $p<.01)$ et moins adaptée aux occasions gaies $(3,64$ contre 4,21 pour le GC ; $F=3,52 ; p<.05$ ) et, d'autre part, sur le degré d'adaptation au secteur des jouets : la marque est moins adaptée $(2,74$ contre 3,62 pour le $\mathrm{GC} ; F=5,65 ; p<.05)$. Cette influence n'est pas enregistrée pour le programme triste.

\section{DISCUSSION GÉNÉRALE ET NOUVELLES HYPOTHESES}

L'expérimentation apporte donc des supports en faveur du modèle de la mésattribution de la familiarité. Dans ce cadre expérimental, nous réfutons le modèle du transfert sémantique automatique (tableau 10). Les effets de l'opération de parrainage TV sont puissants pour le programme gai : une semaine après, deux uniques expositions à la séquence de la nouvelle marque ont des effets significatifs sur plusieurs variables. L'action a donc agi en faisant engrammer (mémoriser) de nouvelles représentations. Elle a provoqué la formation de traces mnésiques et une attitude à l'égard de la marque. L'action de parrainage du programme triste n'a eu aucun effet par comparaison à des sujets non exposés. Nos hypothèses, concernant la première étape uniquement, ont donc sous-évalué les effets des valences des programmes sur les impressions de familiarité. L'explication des différences d'effets entre les actions de parrainage des programmes gai et triste est difficile compte tenu de l'existence d'au moins trois types d'impacts de la valence affective des programmes. Lorsque le téléspectateur ressent des émotions, elles peuvent, par comparaison à une apparition sans émotion, soit accentuer les effets, soit influer négativement, soit avoir les mêmes effets ${ }^{20}$. Or, le principe de la comparaison statistique des effets, à chaque fois par paire de groupes, ne permet pas d'isoler les réels impacts : un même résultat peut s'expliquer par, au moins, deux modes d'influence (un par programme). Deux types de modèles de processus sont proposés : des hypothèses relatives aux valences des programmes et des états affectifs, d'une part et, une hypothèse relative à l'intensité des états affectifs d'autre part (figure 2).

\section{Les effets de la valence négative du programme sur les variables de marque}

Trois hypothèses d'action sont présentées selon un ordre décroissant de probabilité :

- Des effets perturbants au cours de la réception de l'action : l'influence néfaste de l'état affectif désagréable

La première hypothèse explique l'absence d'effet de l'action du programme triste par la présence de

20. De nombreux supports expérimentaux existent en psychologie : Hesse et Spies (1996), Forgas (1994), Mackie et Worth (1991), Isen (1987).

Tableau 10. - Conclusions concernant les deux modèles testés

\begin{tabular}{|l|l|c|}
\hline & \multicolumn{1}{|c|}{ Hypothèses } & Conclusions \\
\hline H1 et H2: & $\begin{array}{l}\text { Présence d'une impression de familiarité chez tous les exposés, } \\
\text { indépendamment de la valence affective du programme parrainé }\end{array}$ & invalidées \\
\hline H3: & $\begin{array}{l}\text { Mésattribution de la familiarité aux évaluations affectives de la marque } \\
\text { et aux attributs marketing }\end{array}$ & preuves en sa faveur \\
\hline H4: & Absence de transfert sémantique automatique & validée \\
\hline
\end{tabular}




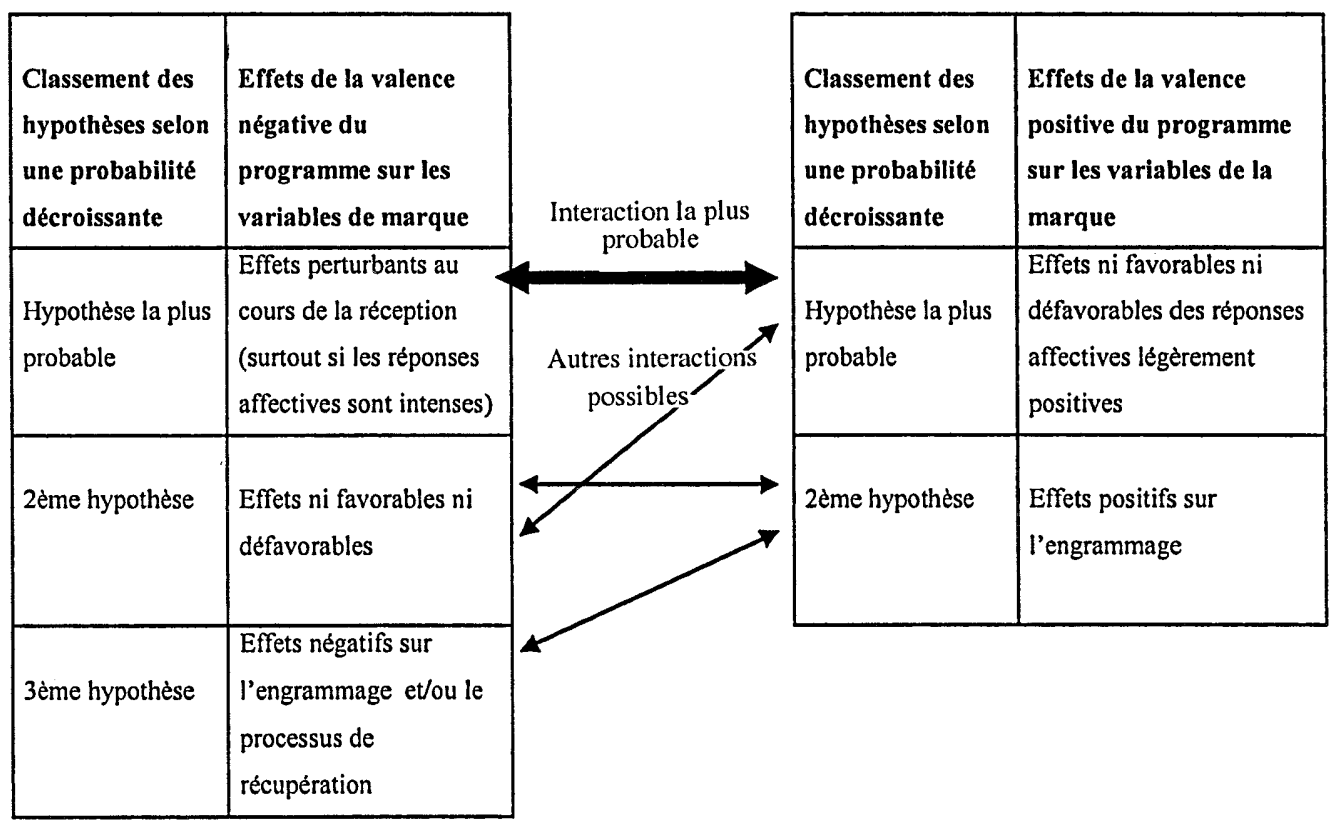

Figure 2. - Hypothèses explicatives sur les interactions entre les effets des valences différentes des programmes parrainés

pensées impertinentes, congruentes à l'état affectif (donc de valence affective négative), pendant la réception de la séquence postgénérique ${ }^{21}$. Elles consomment une partie des ressources limitées de la mémoire de travail. L'attention ne se porte pas sur la séquence : les capacités cognitives ne le permettent pas. Ainsi, pendant les six secondes où la marque apparaît, celle-ci n'est pas traitée de manière élaborée. La seule exposition en prégénérique ne permettrait alors pas, à elle seule, un engrammage des représentations structurales de la marque : aucune impression de familiarité n'est enregistrée une semaine après. Il est donc logique qu'aucun effet ne soit constaté sur les attributs de la marque puisqu'aucune mésattribution n'est possible. Ces hypothèses se rapprochent des conclusions de Seibert et Ellis (1991). Dans notre expérience, il faut allouer de l'attention à deux séquences au moins pour pouvoir observer les premières traces mnésiques.

21. Les contenus de ces pensées impertinentes peuvent être variables : le sujet peut, par exemple, repenser au programme, lutter contre l'instauration chez lui d'un sentiment de tristesse (mécanisme de défense contre l'angoisse)...

\section{- Des effets ni favorables ni défavorables de l'état affectif négatif}

La seconde hypothèse explique la différence d'effets par l'unique action du programme gai. Deux uniques expositions, en présence d'un programme de valence neutre ou de valence triste, ne seraient pas suffisantes pour engrammer la marque-parrain. Seule l'association avec un programme gai le permettrait. Les émotions tristes n'auraient pas eu d'effet réellement perturbant. Cette explication serait cependant en contradiction avec les résultats obtenus par les expériences sur la simple exposition (mère exposure) : deux expositions auraient dû provoquer un effet sur les évaluations affectives une semaine après (voir Bornstein, 1989).

\section{- Des effets négatifs sur l'engramme et/ou le processus de récupération}

La troisième hypothèse explique l'absence d'impact par un effet d'engrammage des émotions négatives : la valence négative serait stockée, selon 
des modalités à déterminer, avec les représentations de la marque. L'état affectif négatif perturberait certains processus :

- il supprimerait les effets de simple exposition : il faudrait pour tester cette hypothèse fabriquer un programme de valence affective réellement neutre ${ }^{22}$. Seule une comparaison avec les effets d'une action de parrainage d'un tel programme permettrait de tester cette hypothèse ;

- il engrammerait l'émotion négative avec la marque. Cette hypothèse, chère aux psychanalystes, a été avancée dans le cadre des travaux plus scientifiques sur l'encodage contextuel (voir Tulving, 1983, 1985);

- il freinerait la récupération de la marque en mémoire : celle-ci serait engrammée mais plus difficilement récupérable dans certaines conditions.

\section{Les effets de la valence positive du programme sur les variables de marque}

En parallèle des effets du programme triste, le programme gai a lui-même ses propres effets. Deux hypothèses sont proposées.

\section{- Des effets ni favorables ni défavorables de l'état affectif légèrement positif}

La mésattribution ne se ferait pas systématiquement à des variables d'évaluation affective mais également à d'autres variables (hauteur de la gamme, secteurs de produit, occasions de consommation). La nature des variables sur lesquelles se porte la mésattribution se ferait selon un mécanisme qu'il faudrait analyser. Cette hypothèse est susceptible de remettre en cause les conclusions de Zajonc (1980, 1984). Nos résultats montrent, en effet, que davantage d'informaticiens exposés au programme gai ont une impression de familiarité. Pourtant, on n'observe pas d'augmentation des scores d'évaluation affective positive. L'impression de familiarité apparaît avant que le sujet mette en route le processus d'évaluation affective. En suivant notre hypothèse, seule la variable « hauteur de gamme des produits » a fait l'objet de cette

22. Ce qui nous semble difficilement envisagable : toute exposition déclenche des réactions ou des évaluations affectives. mésattribution pour le groupe. En outre, pour aller dans ce même sens, tous les items « plaisant $v s$ déplaisant " sont non significatifs. Or, d'après la théorie de la simple exposition, l'impact n'a lieu que sur cette variable affective. D'un autre côté, les variables mesurant la familiarité montrent, quant à elles, des résultats significatifs : elles sont sensibles à l'exposition. Les conclusions de l'expérience seraient expliquées essentiellement par une influence négative de la valence triste du programme. L'action de parrainage du programme gai serait alors presque identique à une action de parrainage d'un programme qui n'aurait aucune valence affective typique. Ce mécanisme d'influence nous semble le plus probable.

\section{- Des effets positifs sur l'engramme de la marque}

La seconde hypothèse, moins probable, explique l'effet de la valence affective positive du programme par un encodage contextuel interactif de cette valence : cette dernière serait engrammée directement dans la marque (ce type d'encodage est expliqué par Baddeley, 1982) : la hauteur de gamme des produits est alors plus élevée, les occasions de consommation et le secteur (jouets) sont différents. Cette hypothèse est cependant peu solide car les théories sur l'encodage contextuel interactif des émotions ne sont pas véritablement validées (Baddeley, 1992). De plus, les évaluations du programme gai des informaticiens montrent que le film a été jugé plutôt décevant. Les réponses du GE (qui l'a véritablement trouvé amusant) sur les attributs de la marque ne sont pas différentes de celles des sujets non exposés. On observerait plutôt, dans ce cas, un encodage contextuel indépendant (la trace de la valence s'observe non dans le contenu des attributs mais dans les évaluations affectives de la marque ; voir Davies, 1986). Le GE a jugé ce programme amusant et le GI plutôt décevant. Cette différence pourrait s'observer dans les évaluations de la marque : le GE l'apprécie davantage que le GI (par comparaison au GC négatif).

\section{L'hypothèse de l'effet négatif des émotions intenses}

Les mesures des états affectifs montrent que, indépendamment de la valence elle-même, le programme triste est jugé émotionnellement plus fort 
(intense) que le programme gai. Il a pu provoquer une diminution de l'attention et ainsi nuire à l'encodage des éléments de la séquence. Une séquence n'a alors pas été traitée de manière contrôlée : soit aucune trace n'a pu être enregistrée ; soit les traces mnésiques ne sont pas suffisamment accessibles pour être enregistrées avec nos outils une semaine après.

Avec notre expérimentation, il n'est pas possible de savoir si l'hypothèse de l'impact de l'intensité est plus plausible que l'hypothèse de l'impact de la valence négative. Au regard des résultats de Walliser (1994, 1996) en parrainage sportif et Pavelchak, Antil et Munch (1988) en publicité, il semble que l'hypothèse de l'intensité soit plus probable. Les deux processus d'influence peuvent également interagir.

\section{LIMITES ET NOUVELLES PISTES DE RECHERCHE}

Même si nous avons voulu varier les profils d'échantillons, et si la qualité du matériel permettrait sa diffusion sur une chaîne TV, la validité externe n'a pas été directement visée. Pour généraliser les résultats, les recherches expérimentales sur ce thème doivent s'étoffer en augmentant le nombre de répétitions, en variant les publics, le matériel et les conditions de réception. Outre la confiance en la réponse des sujets dans le questionnaire post-expérimental, la validité interne est à évaluer :

- Au niveau de la nature verbale des mesures. Le questionnaire permet difficilement d'observer des micro-processus cognitifs et le moment précis où les variables ont agi : des méthodes d'enregistrement des temps de réaction, utilisées en psychologie cognitive, pourraient véritablement affiner les modèles de processus expliqués dans cet article. D'autres outils comme des tests projectifs où la marque est montrée compléteraient utilement les échelles d'attitude et permettraient de mettre en évidence un éventuel transfert d'autres attributs non sélectionnés ici.

- Sur le critère de la ressemblance des programmes, dans les recherches sur les émotions, l'isolement des variables est toujours problématique. Nous avons tenté de fabriquer deux programmes quasi identiques. Cependant, pour déclencher des émotions contrastés dans chaque groupe, on a dû légèrement modifier certains éléments du contenu. Des relations causales fines sont donc difficiles.

Les perspectives de recherche sont nombreuses. Il conviendrait maintenant de s'intéresser aux effets de l'intensité des émotions indépendamment de la valence (triste ou gaie). Ce dernier exige, cependant, encore de plus larges échantillons : une forte déperdition dans les effectifs a limité nos résultats en faisant disparaître un groupe. L'évolution dans le temps des effets et des traces mnésiques serait également un autre objet d'étude prioritaire.

En quittant la logique des théories de processus, on pourrait introduire de nouvelles variables explicatives et les traiter avec des équations structurelles : les attitudes à l'égard des marques à la TV et du parrainage $\mathrm{TV}$, les différentes implications ${ }^{23}$, la sensibilité aux marques, les niveaux d'attention allouée à la séquence et au programme notamment.

\section{CONCLUSIONET IMPLICATIONS OPÉRATIONNELLES}

L'article étudie les effets expérimentaux du parrainage de deux programmes quasi identiques, mais de valences affectives différentes, sur l'image et les évaluations d'une nouvelle marque. Des mesures indirectes enregistrent automatiquement les traces réelles des actions. Les résultats sont en accord avec le nouveau modèle de la mésattribution de la familiarité : les messages de parrainage TV, même faiblement répétés, pourraient uniquement rendre la marque familière au téléspectateur. Ainsi, sur le lieu de vente, le consommateur ressentirait immédiatement, au contact de la marque, une vague impression de l'avoir déjà vue. Oubliant la source télévisuelle, il mésattribuerait de manière non consciente cette impression à d'autres caractéristiques positives susceptibles de déclencher l'achat. Comme supposé et contrairement à certains adages professionnels, aucune trace d'un transfert sémantique automatique du programme sur l'image de la marque n'est observée.

Le parrainage du programme gai, avec seulement deux séquences, provoque des effets positifs indé-

23. Les travaux de Fourquet (1999) sur l'implication seraient alors d'une grande utilité. 
niables sur la marque (sur l'impression de familiarité, les évaluations affectives et la hauteur perçue de la gamme des produits). Nous avons émis des hypothèses pour expliquer l'absence totale d'effet du parrainage du programme triste : il est plus probable qu'elle soit due à l'intensité des réactions affectives déclenchées plutôt qu'à la valence des émotions ressenties (tristesse) par le téléspectateur. Le programme aurait provoqué des pensées impertinentes empêchant de traiter de manière élaborée la séquence postgénérique et d'engrammer la marque. L'intensité des émotions diminuerait la quantité d'attention allouée au traitement du message de la marque et donc limiterait sa mémorisation. Dans ce cas, pour obtenir les premiers effets, il faudrait que le récepteur traite « sans perturbation » au moins deux séquences signées par le parrain.

Si une future réplication de l'expérience confirme ces résultats, trois types d'implications opérationnelles semblent importantes. Pour choisir le programme à parrainer, d'abord, les praticiens ont donc tout intérêt à analyser les émotions que déclenche le programme. Concernant les mesures d'efficacité des actions de parrainage TV ensuite, deux apports expérimentaux sont à souligner. Au niveau méthodologique, l'expérience a montré l'utilité des tests indirects : ils sont en outre faciles à mettre en œuvre. Quant aux variables à mesurer, il semble que certaines sont plus sensibles que d'autres. Il conviendrait, dès lors, de les mesurer systématiquement. C'est le cas de la notoriété assistée (ressemblant à l'impression de familiarité), l'évaluation affective de la marque (dimension affective de l'attitude à l'égard de la marque) et la hauteur perçue de la gamme des produits.

\section{RÉFÉRENCES BIBLIOGRAPHIQUES}

Baddeley A.D. (1992), La Mémoire humaine, théorie et pratique, Presses Universitaires de Grenoble.

Baddeley A.D. (1982), Domains of Recollection, Psychological Review, 89, 6, 708-729.

Bonis (De) M. (1996), Connaître les émotions humaines, Pierre Mardaga.

Bornstein R.F. (1989), Exposure and Affect: Overview and Meta-analysis of Research, 1968-1987, Psychological Bulletin, 106, 2, 265-289.
Bornstein R.F. et Pittman T.S. (1992), Perception without Awareness, New York: The Guilford Press.

Camus J.F. (1996), La psychologie cognitive de l'attention, Paris : Armand Colin, Masson.

Courbet D. (1999), Puissance de la télévision, stratégies de communication et influence des marques, Préface de J.N. Kapferer, Paris, L'Harmattan (coll. Communication), $473 \mathrm{p}$.

Courbet D. (1997), Conception et influence de la communication des organisations. Modélisation et mesure de l'influence du parrainage télévisuel, Thèse de doctorat en sciences de l'information et de la communication, Université de Lyon 3, Lyon, décembre 1997.

Davies G. (1986), Context Effects in Episodic Memory: a Review, Cahiers de Psychologie Cognitive, 6, 2, 157 174.

Derbaix Ch. (1993), La mesure de l'émotionnel et de l'affectif dans la réception des messages publicitaires, Paris, IREP, $237 \mathrm{p}$.

Derbaix Ch., Gerard Ph. et Lardinoit T. (1994), Essai de conceptualisation d'une activité éminemment pratique : le parrainage, Recherche et Applications en Marketing, $10,2,60$.

Forgas J.P. (1994), The Role of Emotion in Social Judgements: an Introductory Review and an Affect Infusion Model (AIM), European Journal of Social Psychology, 24, 1-24.

Fourquet M.P. (1999), Etude socio-cognitive de la réception de la communication persuasive : l'apport du concept d'implication, Actes des $3^{\text {es }}$ Journées d'études Org\&Co, Société Française des Sciences de l'Information et de la Communication, 3-5 juin 1999, Aix-en-Provence.

Ganassali S. et Didellon L. (1996), Le transfert comme principe central du parrainage, Recherche et Applications en Marketing, 11, 1, 37-48.

Giannelloni J.L. (1990), Contribution à l'étude du mode d'influence de la communication par l'événement, Thèse de doctorat en sciences de gestion, Université de Lille Flandres Artois, Lille.

Giannelloni J.L. (1993), L'influence de la communication par l'événement sur la nature de l'image d'entreprise, Recherche et Applications en Marketing, 8, 1, 5-29.

Hesse F.W. et Spies K. (1996), Effects of Negative Mood on Performance: Reduced Capacity of Changed Processing Strategy, European Journal of Social Psychology, 26, 163-168.

Isen A.M. (1987), Positive Affect, Cognitive Processes and Social Behavior, Advances in Experimental Social Psychology, 20, 203-253.

Jacoby L.L. (1991), A Process Dissociation Framework : Separating Automatic Form Intentional Uses of Memory, Journal of Memory and Language, 30, 513541 .

Jacoby L.L., Toth J.P. et Yonelinas A.P. (1993), Separating Conscious and Unconscious Influences of Memory: Measuring Recollection, Journal of Experimental Psychology: General, 122, 139-154. 
Janiszewski C. et Warlop L. (1993), The Influence of Classical Conditioning Procedures on Subsequent Attention to the Conditional Brand, Journal of Consumer Research, 20, 2, 171-189.

Johnson-Laird P.N. (1983), Mental Models: Towards a Cognitive Science of Language, Inference, and Consciousness, Cambridge: Cambridge University Press.

Lardinoit T. (1996), Étude de l'efficacité du parrainage sportif. Effet modérateur des implications durable et situationnelle pour un sport, Thèse de doctorat en sciences de gestion, Université catholique de Louvain.

Mackie D.M. et Worth L.T. (1991), «Feeling Good, but not Thinking Straight : the Impact of Positive Mood on Persuasion », dans Forgas J. (Ed.), Emotion and Social Judgments, Oxford, England: Pergamon Press, 201219.

McClelland J.L., McNaughton B. et O'Reilly R. (1995), Why There are Complementary Learning Systems in the Hypocampus and Neocortex: Insight from the Success and Failures of Connexionnist Model of Learning and Memory, Psychological Review, 102.

McClelland J.L. et Rumelhart D.E. (1988), Explorations in Parallel Distributed Processing, Cambridge Mass.: Bradford Books.

McCracken G. (1989), Who I the Celebrity Endorser? Cultural Foundations of the Endorsement Process, Journal of Consumer Research, 16, 3, 310-321.

Meulemans T. (1998), L'apprentissage implicite, éditions Solal.

Nicolas S. et Perruchet P. (1998), La mémoire implicite : une vue d'ensemble, Psychologie Française, n ${ }^{\circ}$ 43-1, 3-12.

Stadler M. et Frensch P. (éds), (1998), Handbook of Implicit Learning and Implicit Memory, Thousand Oaks, CA: Sage Publications.
Tulving E. (1983), Elements of Episodic Memory. Oxford: OUP.

Tulving E. (1985), How many Memory Systems are there? American Psychologist, 40, 385-398.

Pavelchak M.A., Antil J.H. et Munch J.M. (1988), The Super Bowl: An Investigation into the Relationship among Program Context, Emotional Experience and Ad Recall, Journal of Consumer Research, 15, 3, 360367.

Perruchet P. et Nicolas S. (1998), L'apprentissage implicite : un débat théorique, Psychologie Française, 43, 1, 13-25.

Perruchet P. (1980), (éd.), Les automatismes cognitifs, Pierre Mardaga.

Seibert P.S. et Ellis H.C. (1991), Irrelevant Thoughs, Emotional Mood States and Cognitive Performance, Memory and Cognition, 19 (5), 507-513.

Walliser B. (1994), L'Efficacité du parrainage sportif au sein de la communication de l'entreprise, Thèse de doctorat en sciences de gestion, Université de Grenoble : École Supérieure des Affaires.

Walliser B. (1996), Le rôle de l'intensité des émotions éprouvées par le téléspectateur dans la mémorisation du parrainage, Recherche et Applications en Marketing, $11,1,5-21$.

Watson D., Clark L.A. et Tellegen A. (1988), Development and Validation of Brief Measures of Positive and Negative Affect: The PANAS Scales, Journal of Personality and Social Psychology, 54, 1063-1070.

Zajonc R.B. (1980), Feeling and Thinking: Preferences Need no Inferences, American Psychologist, 35, 151175.

Zajonc R.B. (1984), On the Primacy of Affect, American Psychologist, 39, 117-123. 


\section{Annexe. - Matériel conçu et réalisé}

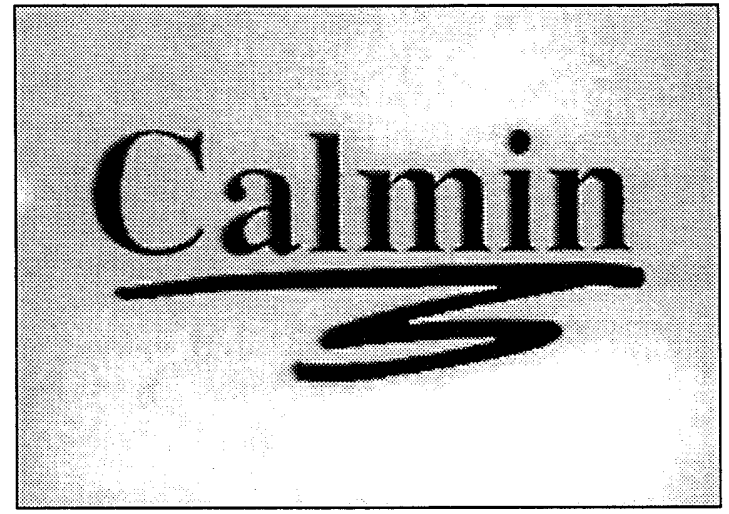

Marque et séquence de parrainage (durée $6 \mathrm{~s}$ )

- Prégénérique avec voix :

«La marque Calmin présente... ».

- Postgénérique avec voix :

«La marque Calmin vous a présenté... ».

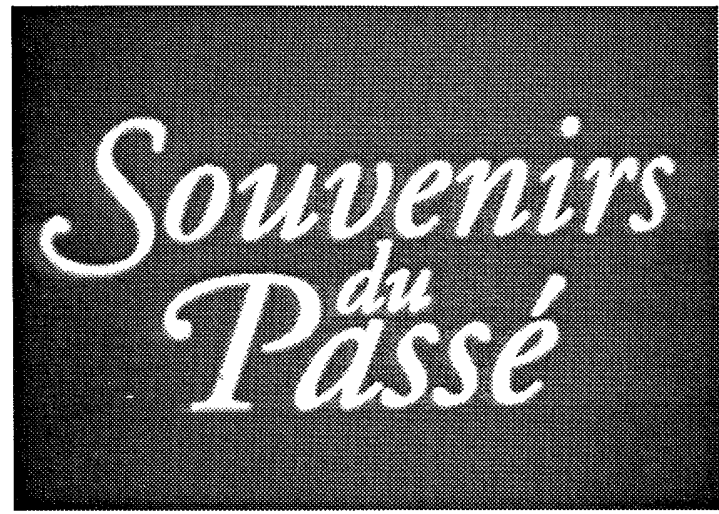

Pré- et postgénériques des programmes (durée 11 s)

Voix : «Souvenirs du passé ».

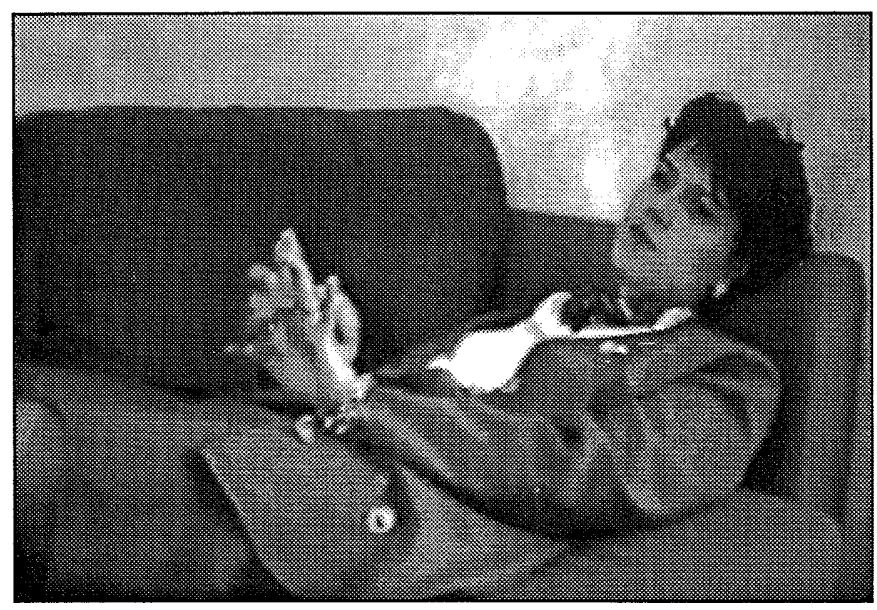

Champ des deux programmes :

«la patiente chez le psychologue»

(durée des deux programmes : $4 \mathrm{mn} 03 \mathrm{~s}$ ). 
Copyright of Recherche et Applications en Marketing is the property of AFM C/o ESCP-EAP and its content may not be copied or emailed to multiple sites or posted to a listserv without the copyright holder's express written permission. However, users may print, download, or email articles for individual use. 